\title{
Complex doping of group 13 elements In and Ga in caged skutterudite
}

\section{$\mathrm{CoSb}_{3}$}

Lili Xi ${ }^{\mathrm{a}}$, Yuting Qiu ${ }^{\mathrm{a}}$, Shan Zheng ${ }^{\mathrm{a}}$, Xun Shi ${ }^{\mathrm{a}}$, Jiong Yang ${ }^{\mathrm{b}}$, Lidong Chen ${ }^{\mathrm{a}}$, David J. Singh ${ }^{\mathrm{c}}$, Jihui

$$
\text { Yang }^{\mathrm{b}^{*}} \text {, Wenqing Zhang }{ }^{\mathrm{a}^{*}}
$$

${ }^{a}$ State Key Laboratory of High Performance Ceramics and Superfine Microstructure, Shanghai Institute of Ceramics, Chinese Academy of Sciences, 1295 Dingxi Road, Shanghai 200050, China

${ }^{\mathrm{b}}$ Materials Science and Engineering Department, University of Washington, Seattle,

$$
\text { WA 98195-2120, USA }
$$

'Oak Ridge National Laboratory, Oak Ridge, Tennessee 37831-6056, USA

The complex doping behavior $\mathrm{Ga}$ and $\mathrm{In}$ in $\mathrm{CoSb}_{3}$ has been investigated using ab initio total-energy calculations and thermodynamics. The formation energies of void filling, Sb-substitution, and complex dual-site occupancy defects with different charge states, and their dependence on chemical potentials of species were studied. Results show that Ga predominantly forms dual-site $2 \mathrm{Ga}_{\mathrm{VF}}-\mathrm{Ga}_{\mathrm{Sb}}$ defects and substitute for Sb only at very high Fermi levels or electron concentrations. In, on the other hand, can play multiple roles in skutterudites, including filling in the crystalline voids, substituting for $\mathrm{Sb}$ atoms, or forming dual-site occupancy, among which the fully charge-compensated dual-site defects $\left(2 \mathrm{In}_{\mathrm{VF}}-\mathrm{In}_{\mathrm{Sb}}\right.$ and $\left.4 \mathrm{In}_{\mathrm{VF}}-2 \mathrm{In}_{\mathrm{Sb}}\right)$ are dominant. The equilibrium concentration ratio of impurities at void-filling sites to those at Sb-substitution sites for Ga-doped $\mathrm{CoSb}_{3}$ is very close to be $2: 1$, while this value markedly deviates from $2: 1$ for In-doped $\mathrm{CoSb}_{3}$. The 2:1 ratio of Ga-doping in $\mathrm{CoSb}_{3}$ leads to low electron concentration $\left(\sim 2 * 10^{19} \mathrm{~cm}^{-3}\right)$ and makes the doped system a semiconductor.

Key words: Defect; Thermoelectric; Ab initio calculations; Skutterudites *Corresponding authors: wqzhang@mail.sic.ac.cn (Wenqing Zhang), jihuiy@uw.edu (Jihui Yang) 


\section{Introduction}

There has been great progress in filled-skutterudite thermoelectric (TE) materials in recent years [1,2]. Studies on filling fraction limits (FFLs) for various impurities in skutterudite $\mathrm{CoSb}_{3}$ are important not only for understanding the transport properties but also for predicting novel systems and compositions. The FFLs for rare-earths (REs), alkaline-earths (AEs), and alkaline metals (AMs) single-element-filled skutterudites have been studied using the density functional ab initio methods and the results show good agreement with experiments [3-15]. A simple electronegativity rule

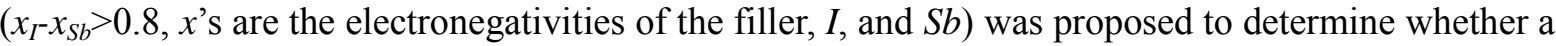
filler impurity can be stably filled into the crystal voids [3]. This rule has predicted ultra-high FFLs for certain $\mathrm{AMs}$ in $\mathrm{CoSb}_{3}$, and these have been confirmed experimentally [13-15]. FFLs in multiple-element-filled $\mathrm{CoSb}_{3}$ have also been investigated [16,17]. The calculated and measured FFLs are in good accord for these electropositive filling elements. In any case, the effect of filling with these elements includes electron-doping, partially compensated by defects, strong thermal conductivity reduction, and modification of the band structure including opening of the gap [18], though not by enough to completely eliminate bipolar effects at low doping [19]. This last fact among others suggests that further optimization is possible leading to still higher ZT.

Interestingly, there are elements that can be incorporated in skutterudites but do not satisfy this electronegativity rule, including Ga and In. Specifically, based on electronegativity one would predict that these elements are not stable fillers in $\mathrm{CoSb}_{3}$, there are several reports that $\mathrm{Ga}$ and In can be doped into the $\mathrm{CoSb}_{3}$ structure. Furthermore, it is reported that these elements affect the electrical and thermal transport properties, and that these doped skutterudites even show improved TE performance. Harnwunggmoung et al. investigated Ga-filled $\mathrm{CoSb}_{3}$ and results showed that only a 
small amount of Ga can be filled into the crystal voids [20]. He et al. and Mallik et al. reported the In-filled skutterudites, seemingly showing a FFL of 0.22 [21-23]. Grytsiv et al. investigated the phase equilibria of In-doped $\mathrm{CoSb}_{3}$, and the maximum solubility of In in skutterudites was reported to be 0.22 when the compound is in equilibrium with $\mathrm{CoSb}_{2}$ and InSb, while the solubility limit is reduced to 0.09 when it coexists with $\mathrm{Sb}$ [24]. The maximum reported thermoelectric figure of merit $\mathrm{ZT}$ value of single In-filled $\mathrm{CoSb}_{3}$ is 1.2 at $575 \mathrm{~K}$ [21]. There have also been reports of double-filled $\mathrm{CoSb}_{3}$ with In. These give FFLs of In in $\mathrm{CoSb}_{3}$ of less than 0.2 and ZT values of 1.43, 1.4, and 1.3 for In-Ce, In-Yb, and In-Ba double filled $\mathrm{CoSb}_{3}$ at $800 \mathrm{~K}$ respectively [25-27]. Recently Sesselmann et al. found that besides the void sites, In can also occupy the Sb sites [28]. In contrast, electropositive elements such as AM, AE and RE clearly cannot substitute for Sb. This is in accord with our first-principles calculations and experiments, which showed that Ga and In atoms could occupy both the voids and $\mathrm{Sb}$ sites in $\mathrm{CoSb}_{3}$ and that these two elements behavior differently with respect to carrier doping [29-31].

Here we extend this work to develop understanding of the substitution, filling and doping behavior of these group 13 elements in $\mathrm{CoSb}_{3}$ based on thermodynamic doping theory and energetics obtained from first principles defect calculations. We systematically study the single impurity induced complex doping properties for Ga- and In-containing $\mathrm{CoSb}_{3}$ skutterudites. The formation energies of many complex defects with different structure and charge states are studied. The results show that both Ga and In mainly form complex dual-site defects. The defect concentrations are studied by using the grand canonical ensemble approximation. We find that the carrier concentration of Ga- and In-doped skutterudites should be very low due to the charge compensation by Sb-substitution.

\section{Theoretical methods}


The present calculations were carried out within density functional theory using the projector augmented wave (PAW) method, as implemented in the Vienna ab initio Simulation Package (VASP), and the Perdew-Burke-Ernzerhof generalized gradient approximation (GGA) [32]. The narrow band gap of $\mathrm{CoSb}_{3}$ is reasonably reproduced with this functional, unlike oxides or other common semiconductors where gap corrections or hybrid functionals need to be used for defect calculations. The calculations with $\mathrm{Ga}$ and In doping are carried out on a supercell $(2 \times 2 \times 2$ primitive cell $)$ containing a total of 128 atoms and 8 voids from the $\mathrm{CoSb}_{3}$ plus the additional dopant atoms. A $3 \times 3 \times 3$ Monkhorst-Pack uniform k-point sampling is used for supercell energy calculations, and $15 \times 15 \times 15$ Monkhorst-Pack uniform k-point sampling is used for other compounds. Lattice constants and ion positions were optimized, and the configuration that has the lowest energy is chosen for further analysis. Details are similar to our prior work on related systems [16,17].

\section{Results and discussions}

\subsection{Crystal structures and complex defect structures}

The skutterudite compounds $\mathrm{TMX}_{3}(\mathrm{TM}=\mathrm{Co}, \mathrm{Rh}, \mathrm{Ir}$, and $\mathrm{X}=\mathrm{P}, \mathrm{As}, \mathrm{Sb})$, are body centered cubic materials, spacegroup Im3. The conventional cubic cell contains 8 formula units, for a total of 32 framework atoms and two voids. The TM occupy the $8 c$ sites and the pnictogen atoms X occupy the $24 g$ sites. The voids at the $2 a$ positions are the 12 fold coordinated by $\mathrm{X}$ atoms. It is these sites that can be filled with RE, ME, and AM satisfying the electronegativity selection rule [3]. As mentioned, Ga and In do not satisfy the electronegativity selection rule and furthermore ionic radii of trivalent Ga and In would seem to be too small for these void sites [33]. Thus other sites, including framework substitutions for $\mathrm{Co}$ and $\mathrm{Sb}$, should also be considered. Additionally, more complex compound 
defects such as void filling-substitution complexes are possible. Here we consider a wide range of possible defect structures including void filling, $\mathrm{Co}$ and $\mathrm{Sb}$ substitution, and complex defects containing different numbers of void fillers and substitution atoms. The detailed structures of these defects are described below.

Four of the possible defect types are particularly favorable. The first of these is filling of the void sites (labeled as $\mathrm{M}_{\mathrm{VF}}, \mathrm{M}=\mathrm{Ga}$, In), and the second is the $\mathrm{Sb}$ substitution defect $\left(\mathrm{M}_{\mathrm{Sb}}\right)$. The third and fourth are complex defects, all involving dual-site occupancy of doping atoms but at different ratios.

One has a 1:1 ratio for void-site to Sb-site occupancy (labeled as $\mathrm{M}_{\mathrm{VF}}-\mathrm{M}_{\mathrm{Sb}}$ ), and the other has a ratio of 2:1 (labeled as $2 \mathrm{M}_{\mathrm{VF}}-\mathrm{M}_{\mathrm{Sb}}$ ). The other complex defects can be obtained by combining these four defects. Figure 1 depicts the stable structures of these four defects. Note that these defects may show different charge states as discussed below. The simple void filling, $\mathrm{M}_{\mathrm{VF}}$ and the $\mathrm{Sb}$ substitutional, $\mathrm{M}_{\mathrm{Sb}}$ have only one configuration. However, for those defects with dual-site occupancy, there are several possible configurations due to the different occupations of $\mathrm{M}_{\mathrm{VF}}$ and $\mathrm{M}_{\mathrm{Sb}}$ and thus varying distances between doping atoms. Based on the total energy calculations, we find that the complex defect has the lowest energy when the distance between the Sb-substitution impurity $\mathrm{M}_{\mathrm{Sb}}$ and the void-filling impurity $\mathrm{M}_{\mathrm{VF}}$ is minimized (Fig. 1c), regardless of the charge states of the defects. This is reasonable both because of the Coulomb interactions between the negative acceptor-like $\mathrm{M}_{\mathrm{Sb}}$ and the positive donor-like $\mathrm{M}_{\mathrm{VF}}$ and also from the point of view of lattice strain ( $\mathrm{Ga}$ and In take space upon void filling, but are smaller than $\mathrm{Sb}$ ). Our calculations show that the Co substitutional, $\mathrm{M}_{\mathrm{Co}}$ defect gives much higher formation energy than the other types of defects. Thus this defect type is excluded and is not considered further. Native point defects in ideal $\mathrm{CoSb}_{3}$ were recently studied by Park et al [34]. Their results showed that all possible native defects in $\mathrm{CoSb}_{3}$ have high positive formation energies, 
among which the Co interstitial $\left(\mathrm{Co}_{\mathrm{i}}\right)$ defect has the lowest. We hence include consideration of $\mathrm{Co}_{\mathrm{i}}$ here.

Skutterudite structure $\mathrm{CoSb}_{3}$ is cubic with equivalent $12 \mathrm{Sb}$ atoms icosahedrally coordinating the void site. However, the symmetry is greatly reduced when defects are added. With one M impurity replacing a $\mathrm{Sb}\left(\mathrm{M}_{\mathrm{Sb}}\right)$, the void distorts and the symmetry is thus reduced. The filler impurity $\left(\mathrm{M}_{\mathrm{VF}}\right)$, shifts away from the center reflecting the small sizes of In and Ga. In the complex defects it moves to a position that is relatively closer to the impurity substituting for $\mathrm{Sb}$ than to other $\mathrm{Sb}$ atoms. The distance of this shift depends on the radius of impurity elements as well as on the chemical bonding between $\mathrm{M}_{\mathrm{VF}}$ and $\mathrm{M}_{\mathrm{Sb}}$. The structure distortion is especially severe for doping with Ga which has a very small size compared both to the available volume of the void and the Sb size. Fig. 2 shows the relaxed positions of several atoms surrounding one specific void site with both the void-filling and Sb-substitution impurities (Ga or In) indicated. It can be observed that $\mathrm{Ga}_{\mathrm{VF}}$ significantly shifts away from the void center and moves very close to the $\mathrm{Ga}_{\mathrm{Sb}}$, leading to a severe distortion of the void and a much shorter $\mathrm{Ga}_{\mathrm{VF}}-\mathrm{Ga}_{\mathrm{Sb}}$ distance comparing to those between $\mathrm{Ga}_{\mathrm{VF}}$ with other $\mathrm{Sb}$ atoms. In fact, the two impurities, $\mathrm{Ga}_{\mathrm{VF}}$ and $\mathrm{Ga}_{\mathrm{Sb}}$, form direct bond with partial covalent character, consistent with the expectation that a complex compound defect may have stable structure due to the intra-defect interaction [29,31]. The complex defect with In doping shows a qualitatively similar distortion pattern, but the shift of $\mathrm{In}_{\mathrm{VF}}$ is small. The underlying reasons are the more spatially extended valence orbitals and larger size of In.

\subsection{Formation energies and relative stabilities of defects}

The formation energies and relative stabilities of the Ga-, and In-related defects in $\mathrm{CoSb}_{3}$ were systematically investigated by ab initio calculations combined with thermodynamics. The approach is 
as described previously $[35,36]$. We mainly focus on $\mathrm{M}_{\mathrm{VF}}, \mathrm{M}_{\mathrm{Sb}}$, and the associated complex compound defects with different charge states, including $\mathrm{M}_{\mathrm{VF}}(0,+1), \mathrm{M}_{\mathrm{Sb}}(0,-1,-2,+1), \mathrm{M}_{\mathrm{VF}}-\mathrm{M}_{\mathrm{Sb}}(0$, $-1,+1), 2 \mathrm{M}_{\mathrm{VF}}-\mathrm{M}_{\mathrm{Sb}}(0,-1,+1), 3 \mathrm{M}_{\mathrm{VF}}-\mathrm{M}_{\mathrm{Sb}}(0,+1), 4 \mathrm{M}_{\mathrm{VF}}-\mathrm{M}_{\mathrm{Sb}}(0,+1,+2), 4 \mathrm{M}_{\mathrm{VF}}-2 \mathrm{M}_{\mathrm{Sb}}(0), 5 \mathrm{M}_{\mathrm{VF}}-2 \mathrm{M}_{\mathrm{Sb}}(0$, $+1)$, and $6 \mathrm{M}_{\mathrm{VF}}-3 \mathrm{M}_{\mathrm{Sb}}(0)$. The numbers in the parentheses indicate the charge states of defects. One specific complex defect, $\mathrm{M}_{\mathrm{Sb}}-\mathrm{Sb}_{\mathrm{VF}}(0,+1,+2,-1)$, is also included to examine the possibility of $\mathrm{M}$ substituting for host atom $\mathrm{Sb}$ accompanied one $\mathrm{Sb}$ occupying the void center. The native defect $\mathrm{Co}_{\mathrm{i}}$ in $\mathrm{CoSb}_{3}$ was also considered, since this was previously reported to be the lowest energy intrinsic defect. We also investigated other defects including $\mathrm{M}_{\mathrm{Co}}$ and $\mathrm{V}_{\mathrm{Sb}}-\mathrm{M}_{\mathrm{Sb}}(\mathrm{V}=$ vacancy), but found them to have relatively high formation energies, and do not discuss them further (see Table 1 for a detailed list).

We use (D, q) to label a defect complex $M_{x} \mathrm{Co}_{32-y} M_{y} S b_{96-z} M_{z}$ with $q$ being the charge state of the defect. The defect formation energy $\Delta G_{f}(D, q)$ per impurity atom can be written as follows $[35,36]$

$$
\begin{aligned}
\Delta G_{f}(D, q)=[ & E_{t o t}(D, q)-E_{t o t}(b u l k)+y \mu_{C o}+z \mu_{S b}-(x+y+z) \mu_{M} \\
& \left.+q\left(\varepsilon_{F}+E_{v}+\Delta V\right)\right] /(x+y+z)
\end{aligned}
$$

where $E_{t o t}(D, q)$ is the total energy of the defect-containing supercell $M_{x} C o_{32-y} M_{y} S b_{96-z} M_{z}$ with doping element $\mathrm{M}$, and $E_{t o t}($ bulk $)$ is the total energy of the defect-free supercell. $x$ is the number of void-filling impurity atoms, $y$ of Co-substitution atoms, and $z$ of Sb-substitution impurities. Here $\mu_{C o}, \mu_{S b}$, and $\mu_{M}$ are the chemical potentials of the different elements. $\varepsilon_{F}$ is the Fermi level referring to the valence band maximum (VBM) $E_{v}$ of the defect-free bulk $\mathrm{CoSb}_{3}$, and $\Delta V$ is the defect-induced correction of the electrostatic potential aligning the valence band maxima (VBM) in all systems [35].

The chemical potentials of $\mathrm{Co}, \mathrm{Sb}$, and impurity $\mathrm{M}\left(\mu_{C o}, \mu_{S b}\right.$, and $\left.\mu_{M}\right)$ in Eq. (1) are dependent 
on experimental environment [37]. These are constrained by condition of thermodynamic equilibrium with stable bulk $\mathrm{CoSb}_{3}$ requiring $3 \mu_{S b}+\mu_{C o}=\mu_{\mathrm{CoSb}_{3}}$; this is the physically meaningful range of the effective chemical potential for element $\mathrm{Sb}$ (or Co) have to satisfy $0 \geq \Delta \mu_{S b} \geq \frac{1}{3} \Delta H_{C_{S S b_{3}}}$ (or $\left.0 \geq \Delta \mu_{C o} \geq \Delta H_{\text {CoSb }_{3}}\right)$, limited by Co-rich $\left(\Delta \mu_{C o} \leq 0\right)$ and Sb-rich $\left(\Delta \mu_{S b} \leq 0\right)$ conditions. $\Delta H_{\text {CoSb }_{3}}$ is the formation enthalpy of $\mathrm{CoSb}_{3}$. The effective chemical potentials for elements are defined by $\Delta \mu_{C o}=\mu_{C o}-\mu_{C o}^{0}$ and $\Delta \mu_{S b}=\mu_{S b}-\mu_{S b}^{0}$, referring to the zero-temperature potentials $\mu_{C o}{ }^{0}$ and $\mu_{S b}^{0}$, i.e. the total energies of bulk $\mathrm{Co}$ and $\mathrm{Sb}$ from ab initio calculations in our model. The calculated formation enthalpy of $\mathrm{CoSb}_{3}$ is $-0.99 \mathrm{eV}$ per formula unit, reasonably agreeing with available experimental data and earlier calculations $[34,38]$. The chemical potential $\mu_{M}$ of impurities is set to be $\mu_{M}=\mu_{M}^{0}$ in estimating $\Delta G_{f}(D, q)$. Experimentally, the value of $\mu_{M}$ should be one that gives absence of both impurity the $M$ and the compound $\mathrm{MSb}(\mathrm{GaSb}, \mathrm{InSb})$ phases. Here we normalize the formation energies on a per impurity atom basis. Thus the $\mu_{M}$ variation within the small range allowed experimentally does not affect the general trends and conclusions of this work. The electronic chemical potential, the Fermi level $\varepsilon_{F}$, also affects doping behavior in $\mathrm{CoSb}_{3}$, but because of the small gap and heavy bands, the range is limited.

Table 1 lists the formation energies of the corresponding zero-charged defects per impurity by using eq. (1). The numbers in the parentheses, such as $2-1,3-2$, and $4-2$, are the abbreviations to denote these complex dual-site occupancy defects, and will be used in the following. The native defects, Co-substitutional defects $\mathrm{M}_{\mathrm{Co}}, \mathrm{M}_{\mathrm{Sb}}-\mathrm{Sb}_{\mathrm{VF}}$, and $\mathrm{V}_{\mathrm{Sb}}-\mathrm{M}_{\mathrm{VF}}$ have much higher formation energies than other defects. The complex dual-site defects with different ratios always have very low formation energies. Therefore, we mainly focus on these complex dual-site defects and two single-element defects $\mathrm{M}_{\mathrm{VF}}$ and $\mathrm{M}_{\mathrm{Sb}}$ for further analysis. 
Fig. 3 shows the formation energies of different defects as a function of the Fermi level for Gaand In-doped $\mathrm{CoSb}_{3}$ in the Co-rich and Sb-rich limits. Here, the zero corresponds to the valence band maximum (VBM). The shaded regions in the figures show the theoretical band gap areas. The configurational entropy $T \Delta S$ contribution to the defect formation energies was treated by assuming a random distribution of impurity atoms at all possible sites with $T$ taken at $1000 \mathrm{~K}$. Each kink in the curves indicates a transition of the corresponding defect types with different charge states.

We find that the dominant defects in both Ga- and In- doped $\mathrm{CoSb}_{3}$ depend on the chemical potentials of $\mathrm{Sb}$ or $\mathrm{Co}$, i.e. experimental synthesis conditions, as well as on the position of the Fermi level, i.e. carrier concentration. For Ga-doped $\mathrm{CoSb}_{3}$, the dominant defect is always the complex dual-site occupancy defect with the ratio of 2:1 over the entire band-gap area for both the Co-rich and Sb-rich conditions. Only at very high Fermi levels, does the single $\mathrm{Ga}_{\mathrm{Sb}}$ defect with a charge state of -2 and the complex defect $\mathrm{Ga}_{\mathrm{VF}}-\mathrm{Ga}_{\mathrm{Sb}}$ with a charge state -1 become more stable than other defects. This is when the electrons in conduction band stabilize the defect-related dangling bonds. Our calculations indicate that In doping leads to a complicated situation. As shown in Fig. $3 \mathrm{~b}$ and d, In can potentially form either a single filling $\left(\mathrm{M}_{\mathrm{VF}}\right)$, or complex dual-site doping defects at different ratios, or Sb-substitutional defects for both the Co-rich and Sb-rich limits. Complex dual-site occupancy defects at different ratios have very close formation energies for In doped $\mathrm{CoSb}_{3}$, which means that all these defects will coexist.

Previous experimental results have shown that Ga prefers to form complex dual-site occupancy defects with a ratio of $2: 1$ in $\mathrm{CoSb}_{3}$, consistent with the above formation energy results. The doping behavior of $\mathrm{In}$ in $\mathrm{CoSb}_{3}$ is complicated based on our results. Experimental results also show that In-related defects usually have more complex behavior than found in Ga-containing $\mathrm{CoSb}_{3}[30]$. 
Based on our calculations, In can fill into the void at low Fermi level, form dual-site occupancy defects with the increasing Fermi level, and become Sb-substitution defect with charge state -2 at very high Fermi levels. For Fermi levels in the middle of the gap, In prefers complex dual-site occupancy defects with different ratios. Our calculations basically indicate that doping behavior of In is very sensitive to experimental conditions in $\mathrm{CoSb}_{3}$. The filling site, $\mathrm{Sb}$-site, and dual-site doping are all realizable depending on synthesis conditions, consistent with the variety of observations and resulting controversy in literature regarding the doping behavior and site preference of In in $\mathrm{CoSb}_{3}$.

\subsection{Defect concentrations}

Here we adopt the grand canonical ensemble thermodynamic model to describe Ga- and In-doping in $\mathrm{CoSb}_{3}$. The details are given in the Appendix A. The defect concentration can be estimated by minimizing the Gibbs free energy of an approximate grand canonical ensemble containing the host $\mathrm{CoSb}_{3}$ and various isolated but complex defects. The theoretical band gap value $(\mathrm{Eg}=0.17 \mathrm{eV})$ is used in determining the Fermi level by requiring overall charge neutrality. Fig. 4 plots the concentration of all possible defects with different charge states and their dependence on $\mathrm{Sb}$ chemical potential in Ga- and In-doping $\mathrm{CoSb}_{3}$. The figures are divided into two regions by the dotted red vertical lines. The left part is for results with the effective chemical potential of impurity atom $(\mathrm{Ga}$, or $\mathrm{In})$ set to $0 \mathrm{eV}$. The right region is for the cases that the effective chemical potentials of impurity atoms are constrained by the formation of secondary phases MSb (M=Ga, In), i.e. $\mu_{S b}+\mu_{M}=\mu_{M S b}$. In experiments, it is very hard to precisely control the effective chemical potential. Typically, in the growth of $\mathrm{CoSb}_{3}$ the ratio of $\mathrm{Sb}$ and $\mathrm{Co}$ is slightly lower than the nominal 3:1 ratio. So the variations of effective chemical potential of $\mathrm{Sb}$ are most likely confined in a small range from the Sb-rich limiting conditions in experiments. The experimental results always fall into 
very small range in the right parts of Fig. 4.

The calculated carrier concentration from the charge neutrality requirement is shown in Fig. 4. The concentration of those charge-compensated compound defects with dual-site occupancy $\left(\mathrm{M}_{\mathrm{VF}}\right.$ : $\left.\mathrm{M}_{\mathrm{Sb}}=2: 1,4: 2,6: 3\right)$ is also plotted for comparison. Our results show that Ga-doped $\mathrm{CoSb}_{3}$ could exhibit weak p-type behavior but only at very low $\mathrm{Sb}$ chemical potentials and that it quickly becomes $\mathrm{n}$-type as the $\mathrm{Sb}$ chemical potential increases. The crossover from $\mathrm{p}$ - to n-type is actually at very low $\mathrm{Sb}$ chemical potential $(-0.28 \mathrm{eV})$. No doubt, achieving such a low value of the Sb chemical potential will require a precise control of $\mathrm{Sb}$ compositions at levels noticeably below the nominal 3:1 (Sb: $\mathrm{Co}$ ratio). This is likely to be very hard to achieve when one considers the narrow synthesis conditions for obtaining the bulk $\mathrm{CoSb}_{3}$ phase. Meanwhile, in almost the whole physical range of $\mathrm{Sb}$ chemical potential, the electron concentration $\left(\sim 2 * 10^{19} \mathrm{~cm}^{-3}\right.$ at $\left.1000 \mathrm{~K}\right)$ is always slightly higher than the hole concentration $\left(\sim 4^{*} 10^{18} \mathrm{~cm}^{-3}\right.$ at $\left.1000 \mathrm{~K}\right)$, leading to the conclusion that the Ga-doping of $\mathrm{CoSb}_{3}$ usually yields n-type behavior. Due to the strong compensation effect among donor-like and acceptor-like defects, the maximum achievable electron concentration is found to be low, less than $2 * 10^{19} \mathrm{~cm}^{-3}$ in theory, indicating that Ga-doping of $\mathrm{CoSb}_{3}$ always yields low electron concentrations. This result is in accord with recent experiments [29].

In-doped $\mathrm{CoSb}_{3}$ shows n-type behavior. The electron concentration is always higher than the hole concentration over the entire achievable Sb chemical potential range. This reflects the fact that In has more probability than $\mathrm{Ga}$ of taking the void filling site. The maximum carrier concentration of In-doped $\mathrm{CoSb}_{3}$ is $4.3 * 10^{19} \mathrm{~cm}^{-3}$ at $1000 \mathrm{~K}$. This value is higher than that of Ga-doped $\mathrm{CoSb}_{3}$, which suggests that In-doped $\mathrm{CoSb}_{3}$ is not completely charge compensated as Ga-doped $\mathrm{CoSb}_{3}$, consistent with recent experiment results [30]. These show that the electron concentrations of In-containing 
skutterudites are indeed higher than those of Ga-containing ones. This further corroborates the conclusion In-containing skutterudites are not fully charge-compensated by defects. The single-filling defect may coexist with dual-site complex defects. The maximum electron concentration (about $2 * 10^{20} \mathrm{~cm}^{-3}$ ) of In-doped $\mathrm{CoSb}_{3}$ in experiments is slightly higher than our theoretical result. This may be because of the complexity of synthesis processes, defect equilibrium at a different temperature than that assumed or possibly non-equilibrium conditions in growth.

The n-type or p-type behavior of the doped compound is dependent on the concentration of different charge defects. As shown in Fig. 4, the complex dual-site occupancy defects are the major defects for both the Ga- and In-doping $\mathrm{CoSb}_{3}$. The completely charge-compensated compound defects especially the 2-1 defects are the dominant ones in Ga-containing $\mathrm{CoSb}_{3}$. The concentrations of other defects, such as the 1-1(-1) and dual-site occupancy defects with ratio of 3-1, 4-1, or 5-2, are much lower. For In-doped $\mathrm{CoSb}_{3}$, the dual-site defects 2-1 and 4-2 are the two dominant defect types, and 1-1(-1) could exist at low Sb chemical potentials. The other defects with different dual-site ratios $(5-2,6-3,4-1,3-1)$ have very similar concentrations. This is consistent with the formation energy results as discussed in relation to Fig. 3. Their concentrations are also substantially lower than that of the dual-site defects 2-1 and 4-2. Interestingly, unlike the Ga-doped $\mathrm{CoSb}_{3}$ with only one type of charge-compensated dual-site 2-1 defect, In-doping leads to the formation of many complex defect clusters with different dual-site occupancy ratios even though their concentrations are lower than those of the 2-1 and 4-2 defects. Except for the charge compensated dual-site defects (4-2, 2-1, 6-3), all the other dual-site defects donate electrons to the systems. This is why the carrier concentration of In-doped $\mathrm{CoSb}_{3}$ is significantly higher than that of Ga-doped system on one hand; and on the other hand, the achievable total concentrations of In impurities are higher than that of $\mathrm{Ga}$ in $\mathrm{CoSb}_{3}$, which 
is also an electron donor defect.

In experiments, one can measure the total concentration of impurities, but it is difficult to quantitatively identify the separate contributions of different defects. Nonetheless, this information is helpful for understanding doping and carrier transport. So we calculate the concentrations of impurities in voids and at the Sb-substitution sites. Fig. 5 shows the atomic concentrations as a function of $\mathrm{Sb}$ chemical potential. Similar to Fig. 4, Fig. 5 is divided into two regions by the vertical lines with different values of impurity effective chemical potential. For the Ga-doped system, the ratio of defect concentrations at void-sites to Sb-substitution sites is very close to 2:1 at both low and high $\mathrm{Sb}$ chemical potential. This means that Ga tends to form dual-site charge-compensated defects. With Sb-rich conditions, the doping limit of $\mathrm{Ga}$ in $\mathrm{CoSb}_{3}$ is 0.12 . This is consistent with the recent experiment results [29]. As Sb chemical potential decreases, the doping levels of $\mathrm{Ga}$ in both the void site and the $\mathrm{Sb}$-site increase. The maximum doping level of $\mathrm{Ga}$ in the void sites is 0.9 at the low $\mathrm{Sb}$ chemical potential limit. Such high doping levels of $\mathrm{Ga}$ in $\mathrm{CoSb}_{3}$ are not found in experiments because the low $\mathrm{Sb}$ chemical potentials are very hard to realize. For the In-doped system, the ratio of concentration of defects in the voids and that at the Sb-substitution sites obviously deviates from 2:1, and the overall defect concentration is higher than that estimated by assuming only the dual-site charge-compensated 2:1 defects, again consistent with the higher electron concentration in both theory and experiments. For the Sb-rich limit, the solubility of $\mathrm{In}$ in $\mathrm{CoSb}_{3}$ is about 0.2 . This value is lower than recent experimental data (0.27) [30]. But when the In solubility is 0.27 , the $\mathrm{Sb}$ compositions in $\mathrm{CoSb}_{3}$ are lower than those of Ga-containing systems. The Sb chemical potential of In-containing $\mathrm{CoSb}_{3}$, when the In solubility is 0.27 is lower than 0 , the value for Ga-containing $\mathrm{CoSb}_{3}$. When chemical potential of Sb marginally departs from the Sb-rich limit, such as $\Delta \mu_{\mathrm{Sb}}=-0.05$ 
$\mathrm{eV}$, the solubility of $\mathrm{In}$ in $\mathrm{CoSb}_{3}$ is 0.272 , and this value is very close to the experimental results. The above results show that the high impurity solubility can be obtained by controlling the chemical potential of Sb, even though this is hard to achieve experimentally. At the low Sb chemical potential limit, the solubility of In in the void site can also reach a very large value (0.7).

The above results mean that group-13 elements Ga and In do not form only the single-filled or $\mathrm{Sb}$-substituted $\mathrm{CoSb}_{3}$ compounds, but rather they enter both the voids and the $\mathrm{Sb}$ sites. This is key to understanding the doping behavior of these additions and also clearly has implications for the thermal conductivity and carrier mobility. Further support for this is given in Fig. 6, which shows a comparison of the theoretically predicted and experimental $\mathrm{Sb}$ composition as a function of the $\mathrm{Ga}$ and In composition. Purely substitutional Ga or In would imply a one to one decrease in Sb content with Ga or In, while void filling would imply no change. Experimentally, we synthesized Ga- and In-doping systems at different conditions. The microscopic compositions were analyzed by different techniques, mainly by SEM and TEM with the nominal composition $\left(\mathrm{Ga}_{\mathrm{VF}}\right)_{0.1} \mathrm{Co}_{4} \mathrm{Sb}_{11.95}\left(\mathrm{Ga}_{\mathrm{Sb}}\right)_{0.05}$. Experimental details are as reported previously [31]. The theoretical predictions for the Ga/In atomic percentage based on the total solubility of $\mathrm{Ga}$ and $\mathrm{In}$ in both void sites and Sb-sites at different $\mathrm{Sb}$ chemical potentials near the Sb-rich limit. The line (black) of $m_{\mathrm{Sb}}=75 \%-5 / 6 n_{\mathrm{M}}(\mathrm{M}=\mathrm{Ga}$, In) in Figure 6 represents the expected relationship between $\mathrm{Sb}$ atomic composition and the concentration of $\mathrm{Ga} / \mathrm{In}$ impurities if the doped system has only the ideally charge-compensated compound defects $2 \mathrm{M}_{\mathrm{VF}}-\mathrm{M}_{\mathrm{Sb}}$. As seen, when the concentration of $\mathrm{Ga}$ or In increases in $\mathrm{CoSb}_{3}$, the $\mathrm{Sb}$ concentration decreases and reasonably follows the behavior that would be expected for fully charge-compensated dual-site defects. Although the measured data are not from single crystals, they are all from single-phase samples. While the agreement is not perfect in that some points deviate 
from the line, the experimental results do support the theoretical conclusion that Ga and In impurities occupy both the void-filling sites and the Sb-substitution sites and form complex dual-site occupancy defects in $\mathrm{CoSb}_{3}$.

\subsection{Possibility of high level Ga/In doping and metastability of defects}

As discussed above, and shown in Fig. 5, Ga/In have high solubility in $\mathrm{CoSb}_{3}$ at low $\mathrm{Sb}_{\text {chemical }}$ potentials. Also as shown in Table I, the defect formation energies are positive at $0 \mathrm{~K}$, which means that the defects are metastable at low temperature. As temperature increases, the configurational entropy plays an important role in stabilizing $\mathrm{Ga} / \mathrm{In}$-containing $\mathrm{CoSb}_{3}$. Fig. 7 shows the formation energies of the charge compensated defects 2-1, 4-2 and 6-3 in $\mathrm{CoSb}_{3}$ at different $\mathrm{Sb}$ chemical potentials. Here the chemical potential of impurities $\Delta \mu_{M}=0$ is used. The contribution of the configurational entropy $T \Delta S$ to defect formation energies is considered at $T=1000 \mathrm{~K}$. The defect formation energy is related to the $\mathrm{Sb}$ chemical potential. For Ga-containing skutterudites, the formation enthalpy of defect $2-1$ has a negative value due to the contribution of configurational entropy, but are positive for the 4-2 and 6-3 defects. Unlike Ga-doped $\mathrm{CoSb}_{3}$, the formation enthalpies are very close to each other and are positive for the three fully charge-compensated defects in In-containing $\mathrm{CoSb}_{3}$. At the low Sb chemical potential limit, the formation energies are very low for all the defects $(2-1,4-2,6-3)$, and for both Ga- and In-containing $\mathrm{CoSb}_{3}$. By controlling the chemical potential of $\mathrm{Sb}$ and considering the contribution of configurational entropy, it is clear that high solubility for Ga and In in skutterudites can be obtained. The reason is that at low Sb chemical potentials, defects that involve Sb deficiency become more favorable.

\section{Conclusions}


We investigated the doping behavior of group 13 elements $\mathrm{Ga}$, In in $\mathrm{CoSb}_{3}$ skutterudites by combining density functional theory and thermodynamics. Incorporation of these elements is energetically disfavored at low temperature. At finite temperature complex behavior involving compensating defects at void filling and Sb substitutional sites is found. The calculated doping behaviors of $\mathrm{Ga}$ and In at finite temperature are dependent on the experimental conditions, and in particular the $\mathrm{Sb}$ chemical potential plays an important role. Both $\mathrm{Ga}$ and In doping are shown to result in complex defects that combine interacting donor-like void-filling and acceptor-like Sb-substitution impurities. The calculated defect formation energies show that Ga can form dual-site doping defects and $\mathrm{Sb}$ substitution defect with charge state -2 , while In forms either void filling, or dual-site doping, or a Sb-substitution defects depending on experiment conditions. For Ga, the concentration ratio in void-site and Sb-site is about 2:1. For In, the concentration ratio departs slightly from 2:1 between the void-sites and the Sb-sites. The ionic radii of Ga and In affect the defect behavior in $\mathrm{CoSb}_{3}$. Our calculations also reveal that Ga-containing $\mathrm{CoSb}_{3}$ always has very low electron concentrations $\left(\sim 2 * 10^{19} \mathrm{~cm}^{-3}\right)$, while the In-containing system can have higher $\mathrm{n}$-type carrier concentrations. The predicted solubility of $\mathrm{Ga} / \mathrm{In}$ in $\mathrm{CoSb}_{3}$ at $\mathrm{Sb}$ rich conditions is consistent with recent experiments. We predict that at Sb-poor conditions they have very high solubility at elevated temperature although the positive formation energies imply that this high defect concentration state is metastable. Turning to thermoelectric applications, as mentioned, even the best n-type multiple-filled-CoSb that has the highest $\mathrm{ZT}$ value may not yet be fully optimized. For example, as mentioned bipolar effects may still be reducing the achievable $Z T$, and of course further thermal conductivity reduction would also be beneficial. The complex behavior of $\mathrm{Ga}$, In impurities in particular suggests that its incorporation in combination with other strategies, in particular rare earth 
filling, may provide needed chemical degrees of freedom for further optimization of the n-type $\mathrm{CoSb}_{3}$ based thermoelectric.

\section{Acknowledgment}

This work is partially supported by National Basic Research Program (973-program) of China under Project No. 2013CB632501, and NSFC Grants (11204333, 11234012, 51121064). Work at ORNL was supported by the Department of Energy, Basic Energy Science through the S3TEC Energy Frontier Research Center.

\section{Appendix A. Grand Canonical Ensemble}

By assuming that the doped system is thermodynamically open and that the formed defects, even with complex defect structure, have no interaction with each other (isolated defect approximation), the grand canonical ensemble can be used to estimate the concentrations of defects.

Given that $\mathrm{N}$ is the number of single-void-containing unit with one lattice void, $12 \mathrm{Sb}$ atoms, and

$4 \mathrm{Co}$ atoms. The unit is to be used as the coarse-grained lattice site to count the configurations of defects. $\mathrm{n}_{\mathrm{i}}$ labels the number of the defect type $i$ existing in the ensemble, and $\mathrm{m}$ is the total number of defect types. The number of defect $\mathrm{Co}_{\mathrm{i}}$ is $\mathrm{n}_{0}$. The number of defects $\mathrm{M}_{\mathrm{VF}}, \mathrm{M}_{\mathrm{Sb}}, \mathrm{M}_{\mathrm{VF}}-\mathrm{M}_{\mathrm{Sb}}$, $2 \mathrm{M}_{\mathrm{VF}}-\mathrm{M}_{\mathrm{Sb}}, \ldots, \mathrm{aM}_{\mathrm{VF}}-\mathrm{bM} \mathrm{M}_{\mathrm{Sb}}$ is labeled as $\mathrm{n}_{1}(1-0), \mathrm{n}_{2}(0-1), \mathrm{n}_{3}(1-1), \mathrm{n}_{4}(2-1), \ldots$, and $\mathrm{n}_{\mathrm{m}}(\mathrm{a}-\mathrm{b})$, respectively. $\mathrm{a}$ and $\mathrm{b}$ are the number of impurities at the void sites and the Sb-substitution sites for a given dual-site occupancy defect. When defect concentration is low, and defect-defect interaction can be neglected, the total Gibbs free energy of the ensemble can be written as:

$J=U-T S-N_{M} \mu_{M}-N_{S b} \mu_{S b}-N_{C o} \mu_{C o}$

Where $U$ is the internal energy, $N_{M}, N_{S b}$, and $N_{C o}$ are the number of $M, S b$, and $C o$ atoms in the 
ensemble, respectively. For the doping system studied in the present work, we have,

$U=E_{0}+\sum_{i=0}^{m} n_{i} \varepsilon_{i}^{\text {def }}, N_{M}=\sum_{i=0}^{m} n_{i} a_{i}, N_{S b}=12 N-\sum_{i=0}^{m} n_{i} b_{i}, N_{C o}=4 N+n_{0}$

$\varepsilon_{i}=E_{i}^{d e f}-E_{0}+q\left(\varepsilon_{F}+E_{v}+\Delta V\right)$

Where $E_{i}^{d e f}$ is the total energy of the supercell with a specific defect $i$, and $E_{0}$ is the total energy of $\mathrm{CoSb}_{3}$ supercell free of defects. By assuming that there is only one defect $\mathrm{M}_{\mathrm{Sb}}$ around one $\mathrm{M}_{\mathrm{VF}}$ in one void, the total configuration entropy of the ensemble can be expressed as

$$
\begin{aligned}
& S=k_{B} \ln \Omega, \Omega=\prod_{i} \Omega_{i} \\
& \Omega_{0}=C_{3 N}^{n_{0}}, \\
& \Omega_{1}(1-0)=C_{N}^{n_{1}}, \\
& \Omega_{2}(0-1)=C_{N}^{n_{2}}\left(C_{12}^{1}\right)^{n_{2}}, \\
& \Omega_{3}(1-1)=C_{N}^{n_{3}}\left(C_{12}^{1}\right)^{n_{3}}, \\
& \Omega_{4}(2-1)=C_{N}^{2 n_{4}}\left(C_{24}^{1}\right)^{n_{4}}, \\
& \ldots \ldots \\
& \Omega_{i}\left(a_{i}-1\right)=\left[C_{N}^{a_{i} n_{i}}\left(C_{12 a_{i}}^{1}\right)^{n_{i}}\right], \\
& \Omega_{i}\left(a_{i}-2\right)=\left[C_{N}^{a_{i} n_{i}}\left(C_{12 a_{i}}^{1}\right)^{n_{i}}\left(C_{12 a_{i}-12}^{1}\right)^{n_{i}}\right], \\
& \Omega_{i}\left(a_{i}-3\right)=\left[C_{N}^{a_{i} n_{i}}\left(C_{12 a_{i}}^{1}\right)^{n_{i}}\left(C_{12 a_{i}-12}^{1}\right)^{n_{i}}\left(C_{12 a_{i}-24}^{1}\right)^{n_{i}}\right]
\end{aligned}
$$

In the condition of thermodynamic equilibrium, the defect concentration can be obtained by minimizing the total Gibbs free energy of the ensemble with respect to the number of defect $i$

$$
\frac{\partial J}{\partial n_{i}}=0
$$

By defining,

$$
\left\{\begin{array}{l}
g_{0}=E_{C o i}^{d e f}-E_{0}+q\left(\varepsilon_{F}+E_{v}+\Delta V\right)-\mu_{C o}, i=0 \\
g_{i}\left(a_{i}-b_{i}\right)=E_{i}^{d e f}-E_{0}+q\left(\varepsilon_{F}+E_{v}+\Delta V\right)-a_{i} \mu_{M}+b_{i} \mu_{S b}, i>0
\end{array}\right.
$$

When $n_{i}<<N$, the concentration of defects is obtained to be 


$$
\begin{aligned}
& C_{0}=n_{0} / N \approx 3 \exp \left(-g_{0} / k T\right) \\
& C_{1}(1-0)=n_{1} / N \approx \exp \left(-g_{1} / k T\right) \\
& C_{2}(0-1)=n_{2} / N \approx 12 \exp \left(-g_{2} / k T\right) \\
& C_{3}(1-1)=n_{3} / N \approx 12 \exp \left(-g_{3} / k T\right) \\
& C_{4}(2-1)=n_{4} / N \approx \frac{\sqrt{24}}{2} \exp \left(-g_{4} /(3 k T)\right) \\
& \ldots . . \\
& C_{i}\left(a_{i}-1\right)=n_{i} / N \approx \frac{\left(a_{i}^{*} 12\right)^{\frac{1}{a_{i}}}}{a_{i}} \exp \left(-g_{i} /\left(a_{i} k T\right)\right) \\
& C_{i}\left(a_{i}-2\right)=n_{i} / N \approx \frac{\left(a_{i} * 12\right)^{\frac{1}{a_{i}}}}{a_{i}}\left(a_{i}^{*} 12-12\right)^{\frac{1}{a_{i}}} \exp \left(-g_{i} /\left(a_{i} k T\right)\right) \\
& C_{i}\left(a_{i}-3\right)=n_{i} / N \approx \frac{\left(a_{i} * 12\right)^{\frac{1}{a_{i}}}}{a_{i}}\left(a_{i}^{*} 12-12\right)^{\frac{1}{a_{i}}}\left(a_{i} * 12-24\right)^{\frac{1}{a_{i}}} \exp \left(-g_{i} /\left(a_{i} k T\right)\right)
\end{aligned}
$$

For an ensemble with many charged defects, the carrier concentration depends on the Fermi level in the following way

$$
n_{e}=N_{C} \exp \left[-\left(E_{g}-\varepsilon_{F}\right) / k_{B} T\right], n_{h}=N_{V} \exp \left(-\varepsilon_{F} / k_{B} T\right),
$$

where $N_{C}$ and $N_{V}$ are the effective density of states of conduction and valence band edges. $E_{g}$ is the band gap of the system.

The Fermi level can be determined by overall charge neutrality, i.e.

$$
n_{e}-n_{h}=\sum_{i} q(i) C_{i} N_{s}
$$

Where $q(i)$ is the charge state of defect type $i . N_{s}$ is the number of sites (per unit volume) at which defect $\mathrm{i}$ can be formed. 


\section{References}

[1] G.A. Slack, New materials and performance limits for thermoelectric cooling, in: D.M. Powe

(Eds), CRC handbook of Thermoelectric, CRC press, Boca Raton, 1995, pp. 407-440.

[2] B.C. Sales, D. Mandrus and R.K. Williams, Science, 272 (1996) 1325-1328.

[3] X. Shi, W.Q. Zhang, L.D. Chen, J. Yang, Phys. Rev. lett. 95 (2005) 185503 and references therein.

[4] L.D. Chen, T. Kawahara, X.F. Tang, T. Goto, T. Hirai, J.S. Dyck, W. Chen, C. Uher, J. Appl. Phys. 90 (2001) 1864-1868.

[5] S.Q. Bai, Y.Z. Pei, L.D. Chen, W.Q. Zhang, X.Y. Zhao, J. Yang, Acta. Mater. 57 (2009) 3135-3139.

[6] Z. Xiong, L.L. Xi, J. Ding, X.H. Chen, X.Y. Huang, H. Gu, W.Q. Zhang, L.D. Chen, J. Mater. Res. 26 (2011) 1848-1856.

[7] J. Yang, W.Q. Zhang, S.Q. Bai, Z.G. Mei, L.D. Chen, Appl. Phys. Lett. 90 (2007) 192111.

[8] X. Shi, H. Kong, C P. Li, C. Uher, J. Yang, J.R. Salvador, H. Wang, L.D. Chen, W.Q. Zhang, Appl. Phys. Lett. 92 (2008) 182101.

[9] X. Shi, J. Yang, J. Salvador, M.F. Chi, J. Cho, H. Wang, S.Q. Bai, J.H. Yang, W.Q. Zhang, L.D. Chen, J. Am. Chem. Soc. 133 (2011) 7837-7846.

[10] X. Shi, S.Q. Bai, L.L. Xi, J. Yang, W.Q. Zhang, L.D. Chen, J.H. Yang, J. Mater. Res. $26(2011) 1745-1754$.

[11] W.Q. Zhang, X. Shi, Z.G. Mei, Y. Xu, L.D. Chen, J. Yang, G.P. Meisner, Appl. Phys. Lett. 89 (2006) 112105.

[12] Z.G. Mei, W.Q. Zhang, L.D. Chen, Phys. Rev. B. 74 (2006) 153202.

[13] Z.G. Mei, J. Yang, Y.Z. Pei, W.Q. Zhang, L.D. Chen, Phys. Rev. B. 77 (2008) 045202.

[14] Y.Z. Pei, L.D. Chen, W.Q. Zhang, X. Shi, S Q. Bai, X.Y. Zhao, Z.G. Mei, X.Y. Li, Appl Phys. Lett. 89 (2006) 221107.

[15] Y.Z. Pei, J. Yang, L.D. Chen, W.Q. Zhang, J. R. Salvador, J.H. Yang, Appl. Phys. Lett. 
95 (2009) 042101.

[16] L.L. Xi, J. Yang, W.Q. Zhang, L.D. Chen, J. Am. Chem. Soc. 131 (2009) 5560-5563.

[17] L.L. Xi, J. Yang, C.F. Lu, Z.G. Mei, W.Q. Zhang, L.D. Chen, Chem. Mater. 22 (2010) $2384-2394$

[18] D.J. Singh, I.I. Mazin, Phys. Rev. B. 56 (1997) R1650-1653.

[19] D.J. Singh, M.H. Du, Phys. Rev. B. 82 (2010) 075115.

[20] Harnwunggmoung, K. Kurosaki, T. Plirdpring, T. Sugahara, Y. Ohishi, H. Muta, S.Yamanaka, J. Appl. Phys. 110 (2011) 013521.

[21] T. He, J.Z. Chen, H.D. Rosenfeld, M.A. Subramanian, Chem. Mater. 18 (2006) 759-762.

[22] R.C. Mallik, J.Y. Jung, S.C. Ur, I.H. Kim, Met. Mater. Int. 14 (2008) 223-228.

[23] R.C. Mallik, E. Mueller, I.H. Kim, J. Appl. Phys. 111 (2012) 023708.

[24] A. Grytsiv, P. Rogl, H. Michor, E. Bauer, G. Giester, J. Electron. Mater. 42 (2013) 2940-2952.

[25] W.Y. Zhao, P. Wei, Q.J. Zhang, C.L. Dong, L.S. Liu, X.F. Tang, J. Am. Chem. Soc.

131 (2009) 3713-3720.

[26] J. Graff, S. Zhu, T. Holgate, J. Peng, J. He, T.M. Tritt, J. Electron. Mater. 40 (2011) 696-701.

[27] H. Li, X.F. Tang, Q.J. Zhang, C. Uher, Appl. Phys. Lett. 94 (2009) 102114.

[28] Sesselmann, T. Dasgupta, K. Kelm, E. Müller, S. Perlt, S. Zastrow, J. Mater. Res. 26 (2011) $1820-1826$.

[29] Y.T. Qiu, L.L. Xi, X. Shi, P.F. Qiu, W.Q. Zhang, L.D. Chen, J.R. Salvador, J.Y. Cho, J.H. Yang, Y.C. Chien, S.W. Chen, Y.L. Tang, G.J. Snyder, Adv. Func. Mater. 23 (2013) 3194-3203.

[30] Y.L. Tang, Y.T. Qiu, L.L. Xi, X. Shi, W.Q. Zhang, L.D. Chen, S.-M. Tseng, S.-W. Chen, G. J. Snyder, Energy Environ. Sci. 7 (2014) 812-819.

[31] Y. T. Qiu, J.J. Xing, X. Gao, L.L. Xi, X. Shi, H. Gu, L.D. Chen, J. Mater. Chem. A. 2 (2014) 10952-10959.

[32] G. Kresse, J. Furthmuller, Phys. Rev. B. 54 (1996) 11169-11186.; G. Kresse, J. Hafner, Phys. Rev. B. 47 (1993) 558-561.

[33] R.D. Shannon, Acta Crystallographica, Section. A. 32 (1976) 751-767.

[34] C. H. Park, Y.S. Kim, Phys. Rev. B. 81 (2010) 085206. 
[35] J. L. Zhao, W.Q. Zhang, X. M. Li, J. W. Feng, and X. Shi, J. Phys: Conden. Matter. 18

(2006) 1495-1508.

[36] R. Nazarov, T. Hickel, J. Neugebauer, Phys. Rev. B. 82 (2010) 224104.

[37]W.Q. Zhang, J.R. Smith, X.G. Wang, Phys. Rev. B. 70 (2004) 024103.

[38]O. Kubaschewski, C.B. Alcok, P.J. Spencer, International Series on Materials

Science and Technology, Materials Thermochemistry, Six edition, Pergamon press, Oxford, 1993, pp. 257-323.

Table 1. Formation energies of different defects at $0 \mathrm{~K}$. Values are given for the chemical potentials in the Co-rich $\left(\Delta \mu_{\mathrm{Sb}}=-0.33 \mathrm{eV}\right)$ and Sb-rich $\left(\Delta \mu_{\mathrm{Sb}}=0 \mathrm{eV}\right)$ limits. The formation energies of the several native defects are also shown in the table for comparison. Only zero charge state defects are listed. The charge state dependence is given in Fig. 3.

\section{Figure Captions}

Fig. 1. Local atomic structures for the four types of defects in M-doped $\mathrm{CoSb}_{3}$, with the defect-related atoms red cycled. (a) Void-filling, $\mathrm{M}_{\mathrm{VF}}$, (b) Sb-substitution, $\mathrm{M}_{\mathrm{Sb}}$, (c) Complex defect with void-filling and Sb-substitution atoms at a 1:1 ratio, $\mathrm{M}_{\mathrm{VF}}-\mathrm{M}_{\mathrm{Sb}}$, (d) Complex defect with void-filling and Sb-substitution complex atoms at a 2:1 ratio, $2 \mathrm{M}_{\mathrm{VF}}-\mathrm{M}_{\mathrm{Sb}}$. Each defect may have different charge states. The green icosahedron indicates void site in the skutterudite lattice.

Fig. 2. Stable configurations of the $\mathrm{M}_{\mathrm{VF}}-\mathrm{M}_{\mathrm{Sb}}$ (1:1) complex defect. The distances between $\mathrm{M}_{\mathrm{VF}}$ (central atom) and some of the 12 neigbor atoms are indicated to illustrate the structure distortion. The figures correspond to Ga- (a) and In-filling (b). The $2 \mathrm{M}_{\mathrm{VF}}-\mathrm{M}_{\mathrm{Sb}}$ (2:1) defect (not shown) has practically the same distances as those indicated in the above figures.

Fig. 3. Defect formation energies (per impurity atom) as a function of the Fermi level at the Co-rich and Sb-rich limits. The defect types are labeled on the figure, with the number on each curve indicating the charge state of the defect.

Fig. 4. Equilibrium defect concentrations for (a) Ga- and (b) In- doped $\mathrm{CoSb}_{3}$ at $1000 \mathrm{~K}$. The defect types are labeled in the figure, with the number in parentheses indicating the charge state of the defect. The calculated concentrations of electrons (n) and holes (p) are also plotted. The figure is divided to two parts by red lines with different chemical potentials of impurity atoms $\mathrm{Ga} / \mathrm{In}$ for the formation of $\mathrm{GaSb}$ or InSb.

Fig 5. Total impurity atomic concentration (with respect to numbers of voids) as a function of $\mathrm{Sb}$ chemical potential for Ga- and In-containing $\mathrm{CoSb}_{3}$ skutterusites. The black lines are the total 
impurity cencentrations. Green lines indicate the total concentration for Ga and In impurities occupying the void sites, and red lines for impurities occupying Sb-substitution sites. The blue lines are the estimated concentrations of impurities at the $\mathrm{Sb}$-substitution sites from the $\mathrm{Ga}_{\mathrm{VF}}$ or $\mathrm{In}_{\mathrm{VF}}$ curves assuming only the dual-site 2-1 defects.

Fig. 6. Theoretical and experimental $\mathrm{Sb}$ atomic percentages as a function of $\mathrm{Ga} / \mathrm{In}$ atomic percentages in Ga- and In-doped $\mathrm{CoSb}_{3}$. Open symbols are experimentally measured results. Solid stars are theoretical data. The statistical composition data from Scanning Electron Microscope (SEM) and Transmission Electron Microscope (TEM) analysis are plotted for the samples with nominal composition $\left(\mathrm{Ga}_{\mathrm{VF}}\right)_{0.1} \mathrm{Co}_{4} \mathrm{Sb}_{11.95}\left(\mathrm{Ga}_{\mathrm{Sb}}\right)_{0.05}$. The black line represents the expected $\mathrm{Sb}$ atomic composition variation as a function of $\mathrm{Ga}$ or In doping based on the ideal defect $2 \mathrm{M}_{\mathrm{VF}}-\mathrm{M}_{\mathrm{Sb}}$.

Fig. 7. Formation energies of a few different fully charge-compensated defects under different $\mathrm{Sb}$ chemical potentials at $1000 \mathrm{~K}$. The lines are guides to the eye to connect the points for related defects. 
Fig. 1.

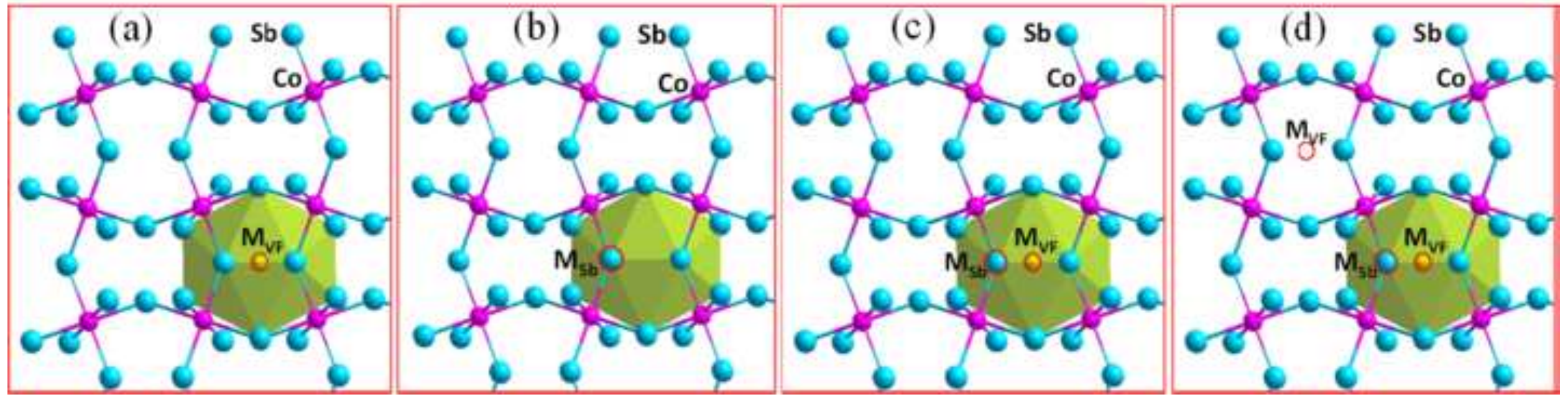


Fig. 2.

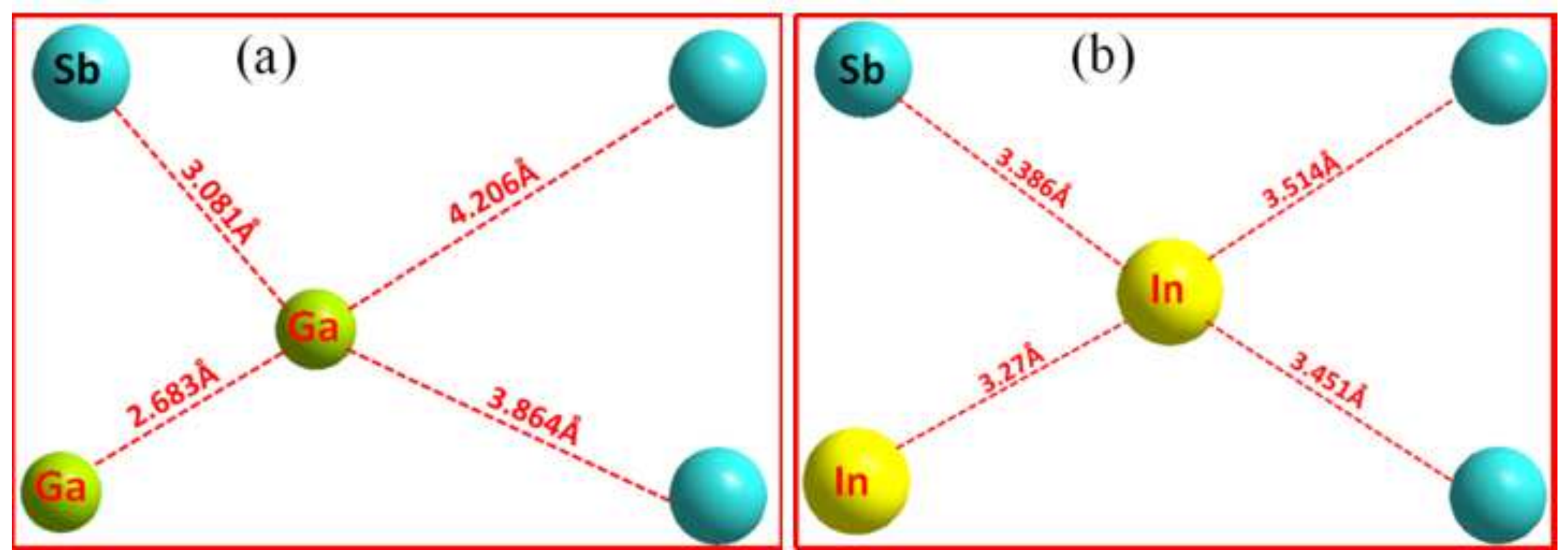


Fig. 3a.

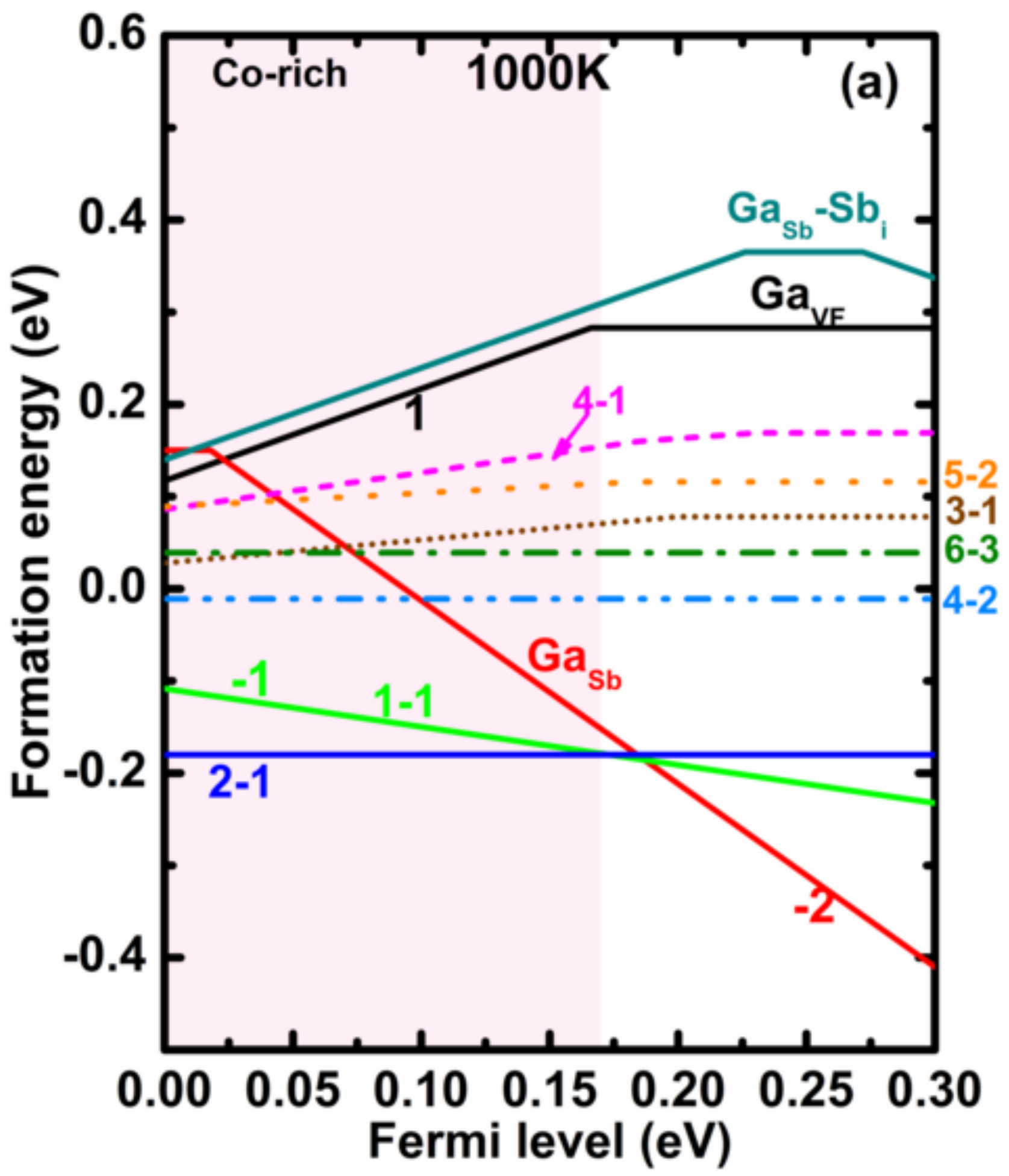


Fig. 3b.

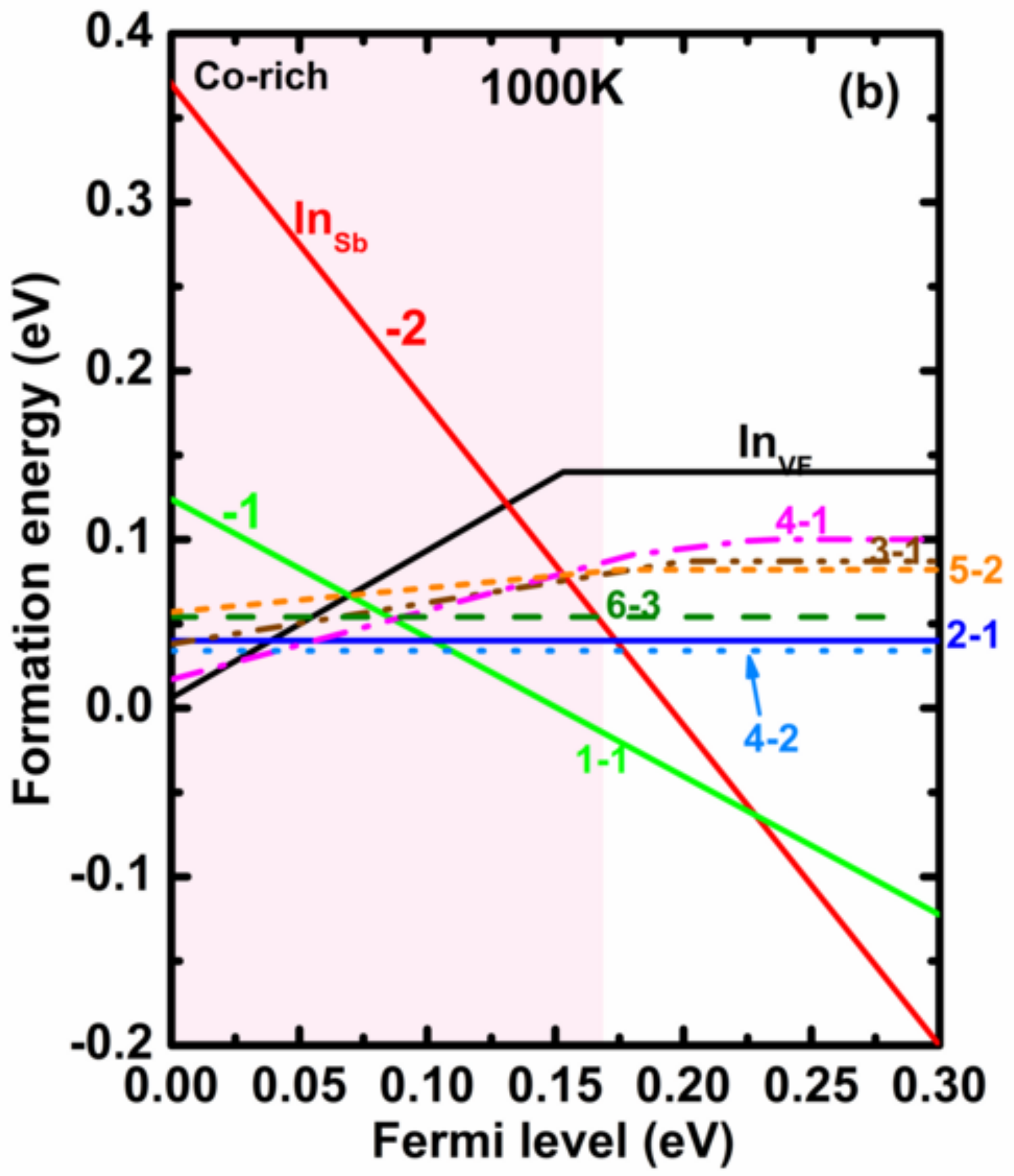


Fig. 3c.

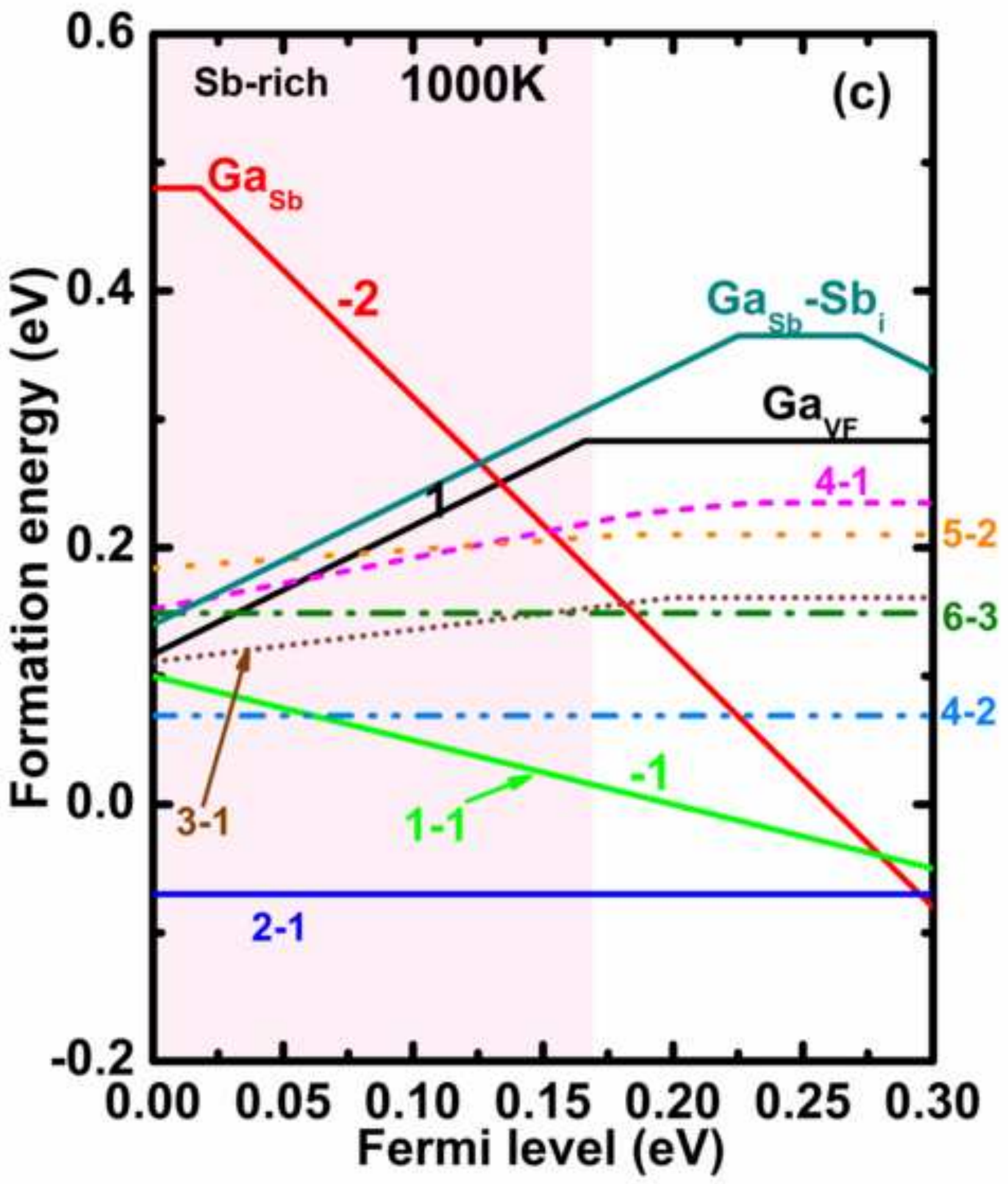


Fig. 3d.

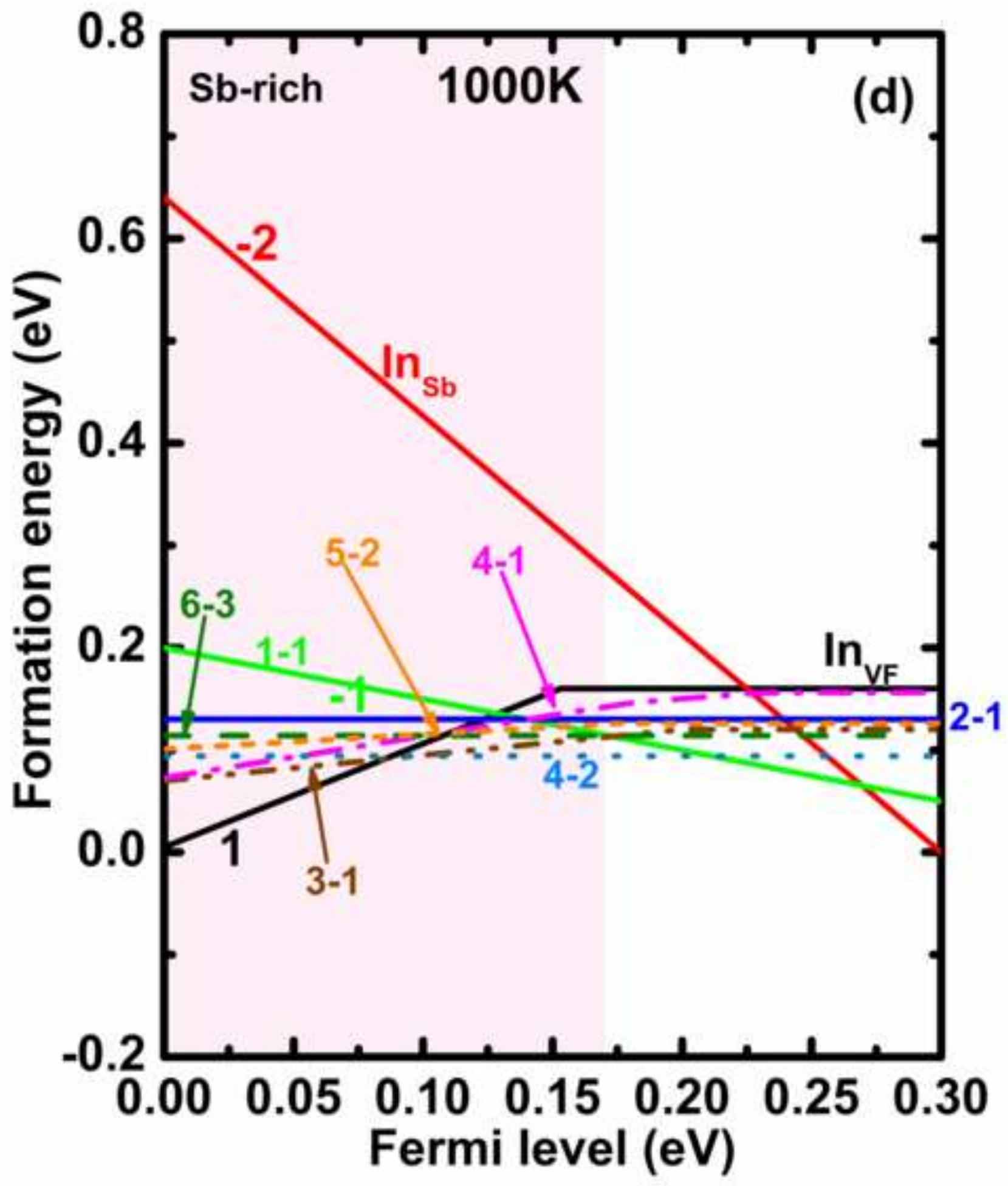


Fig. 4a.

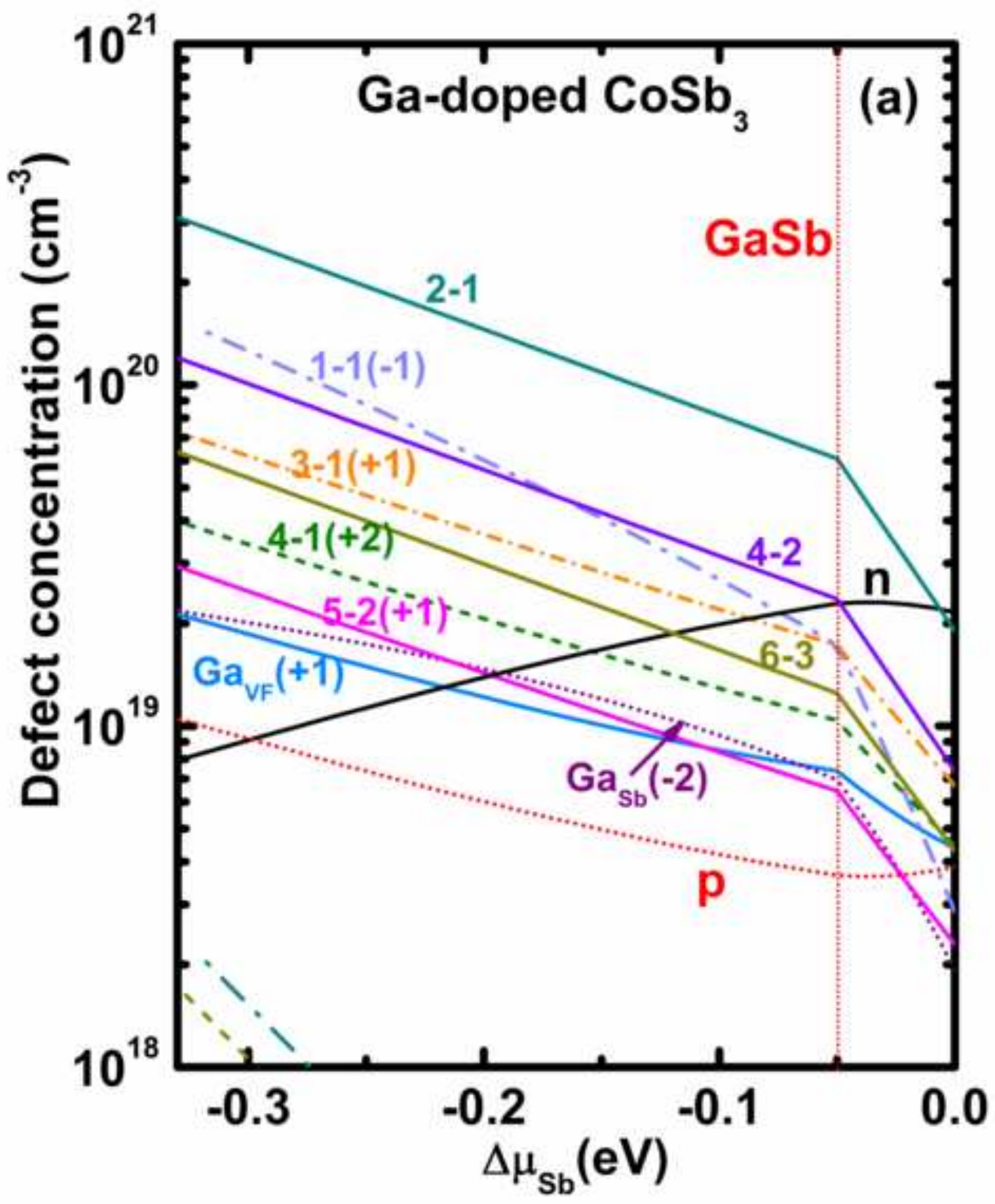


Fig. 4b.

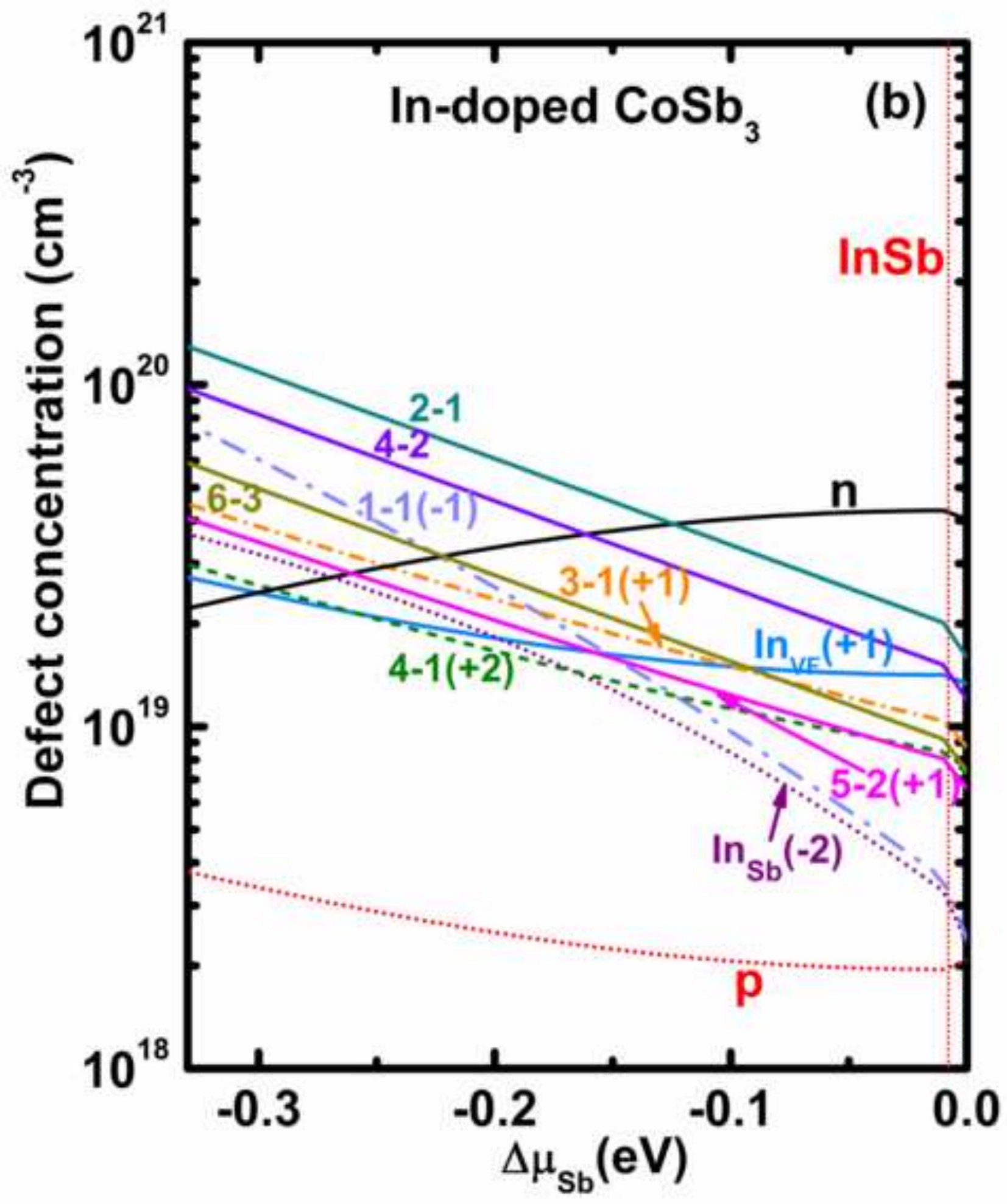


Fig. 5a.

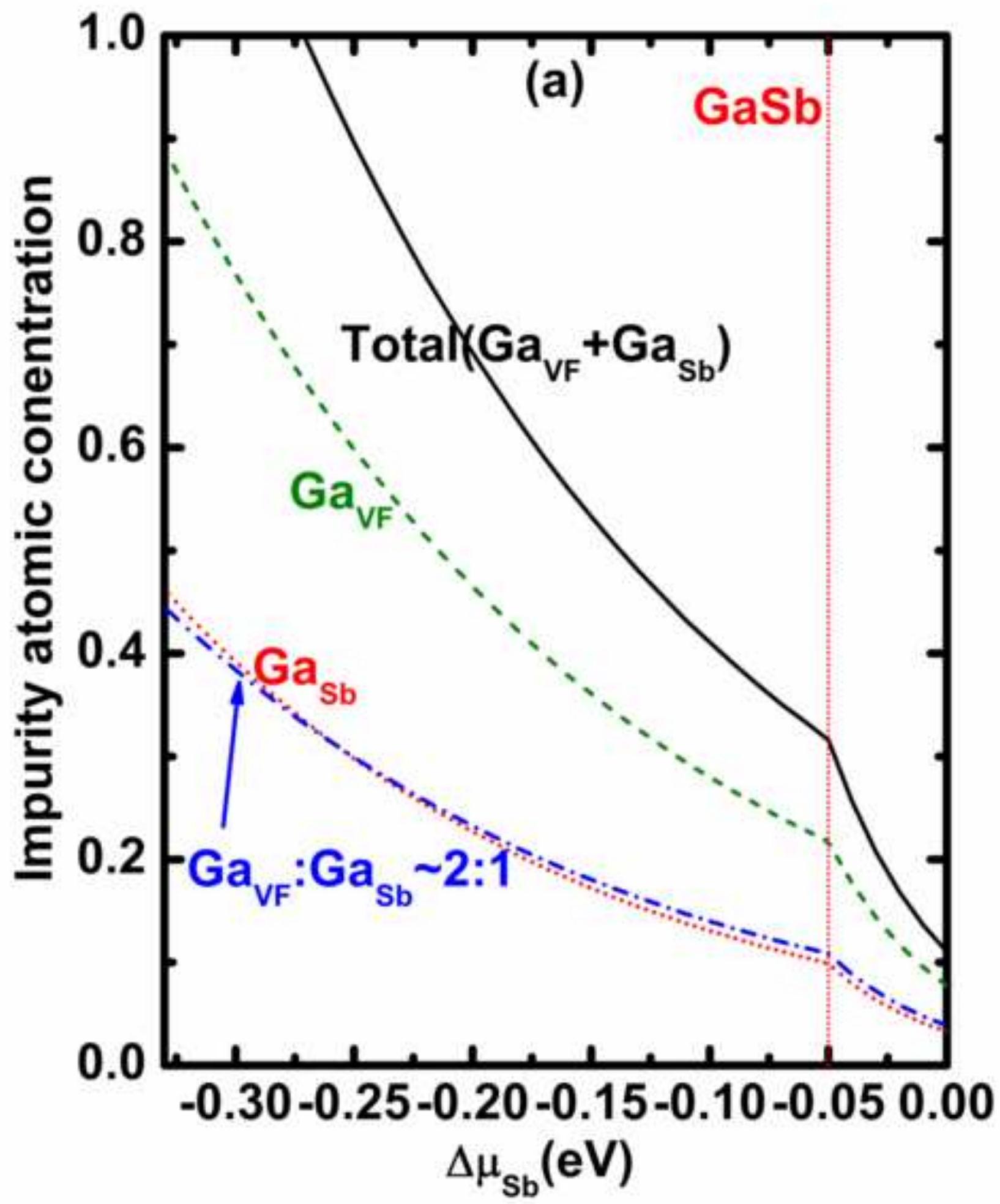


Fig. 5b

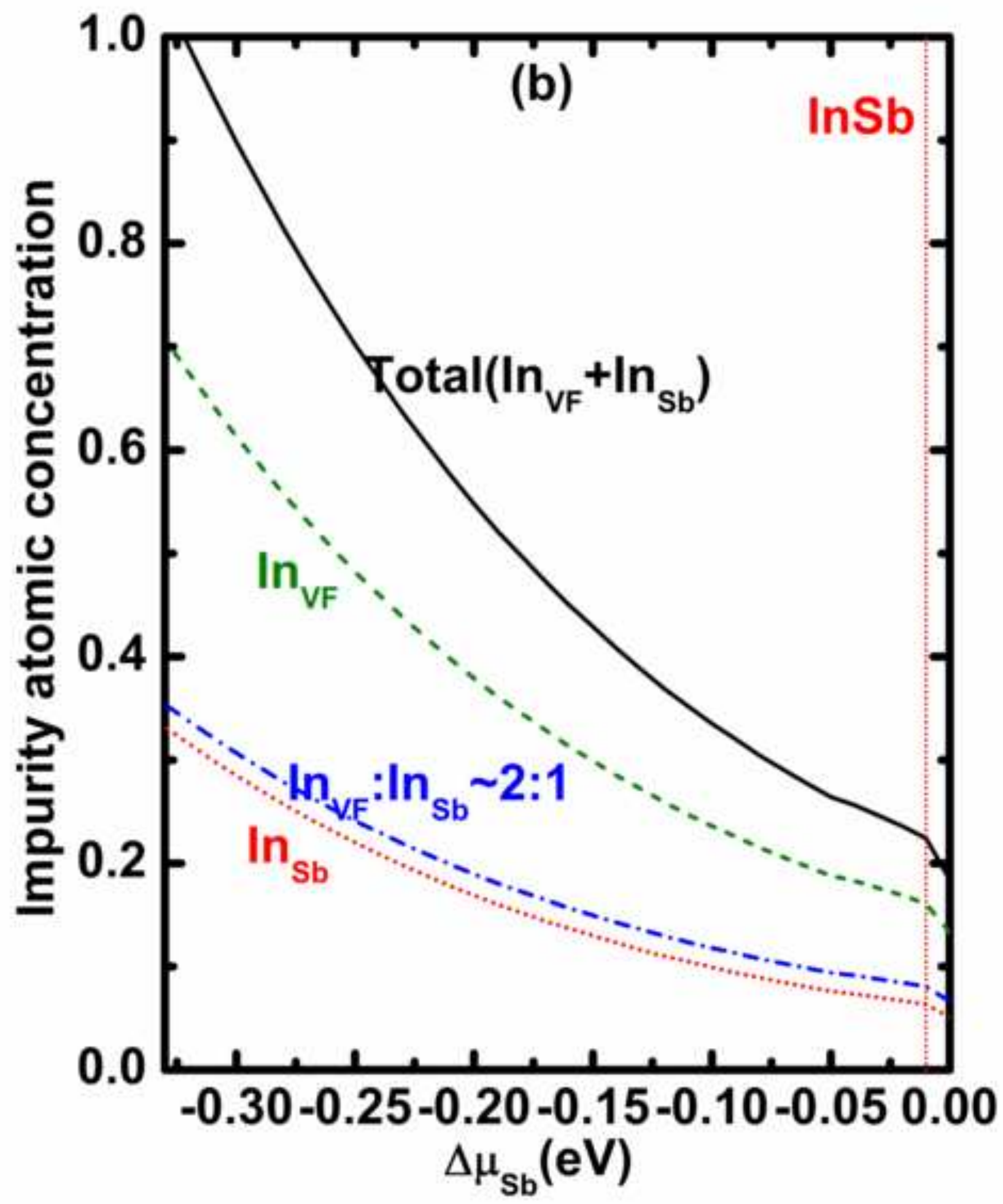




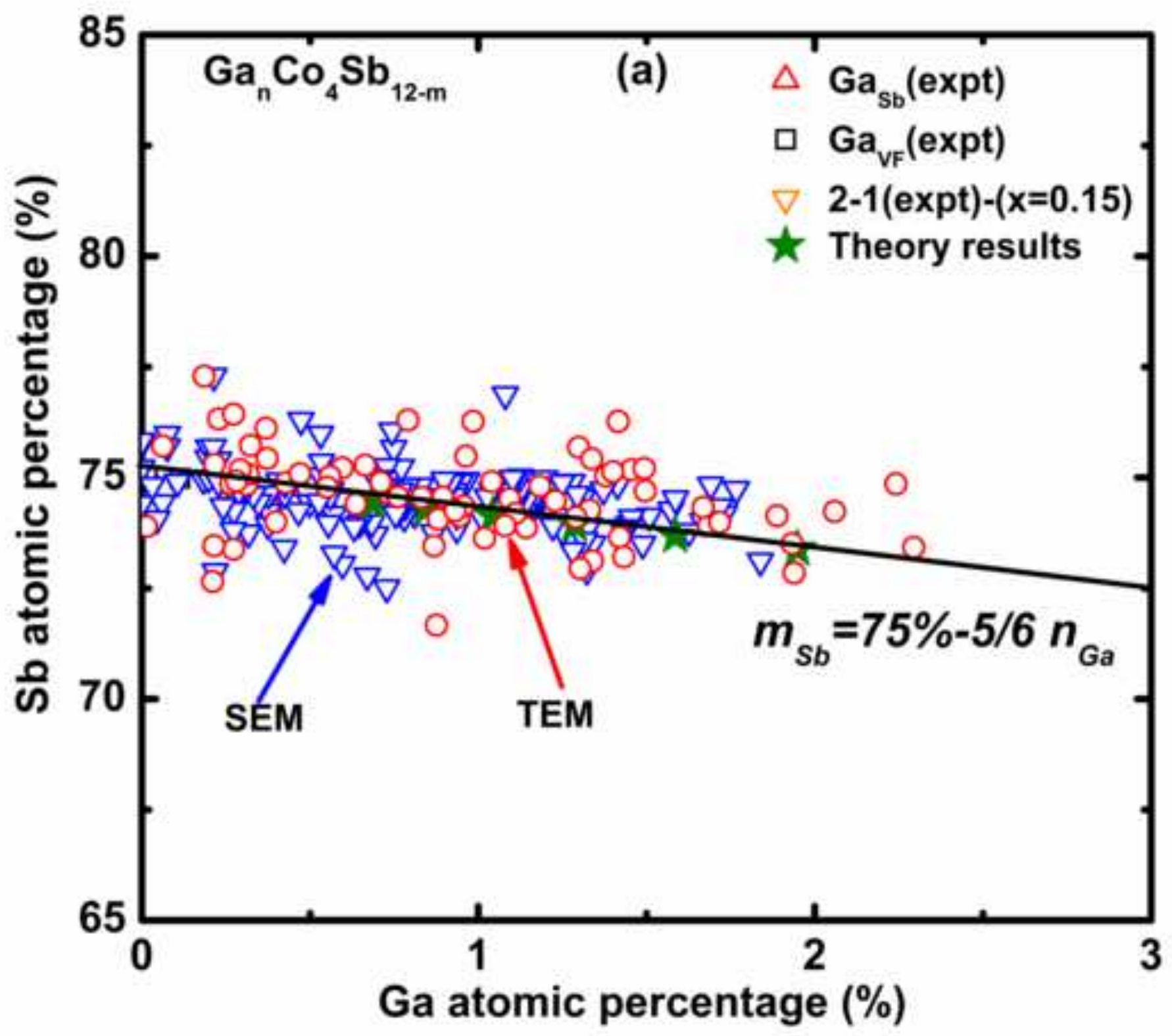




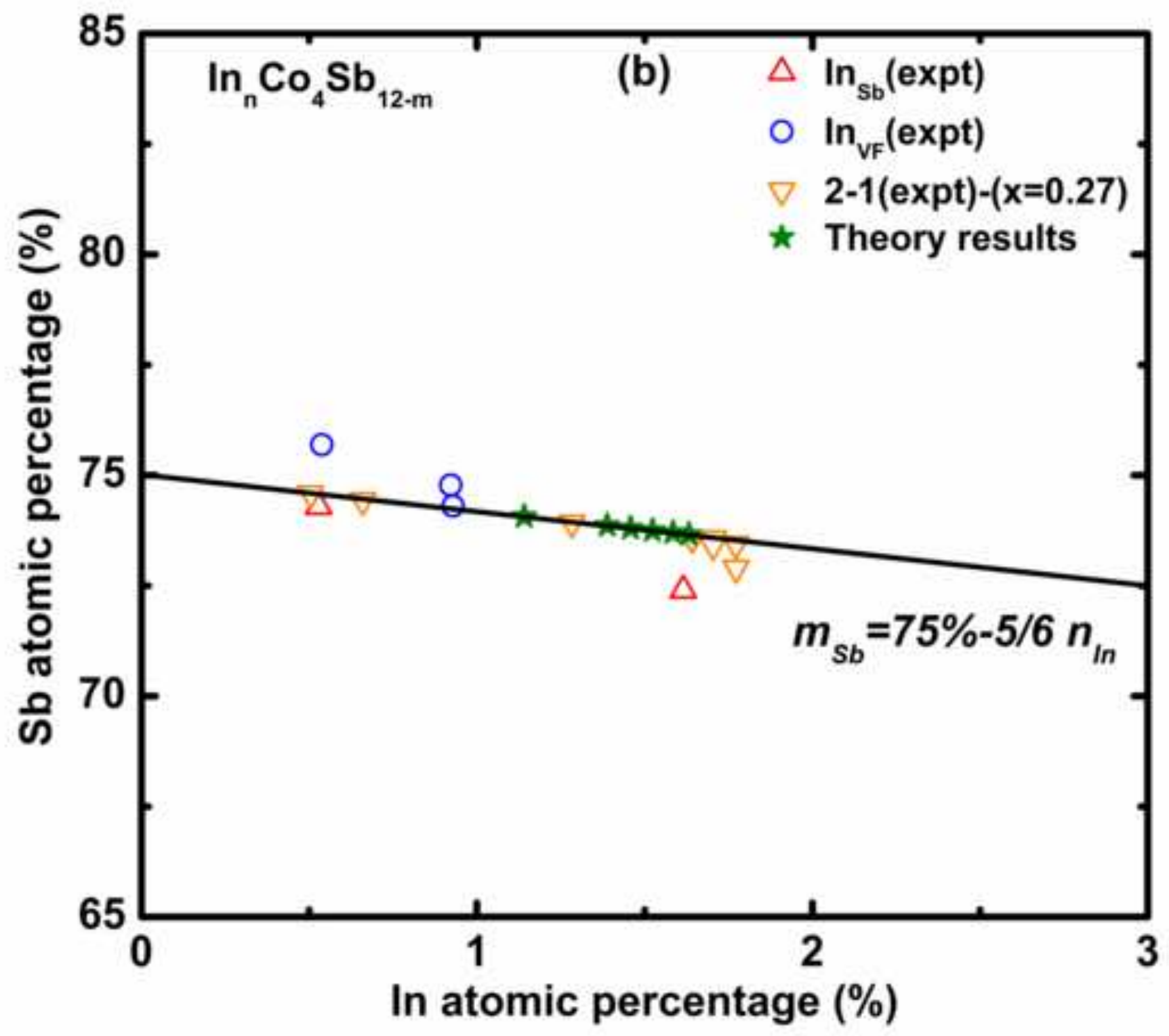




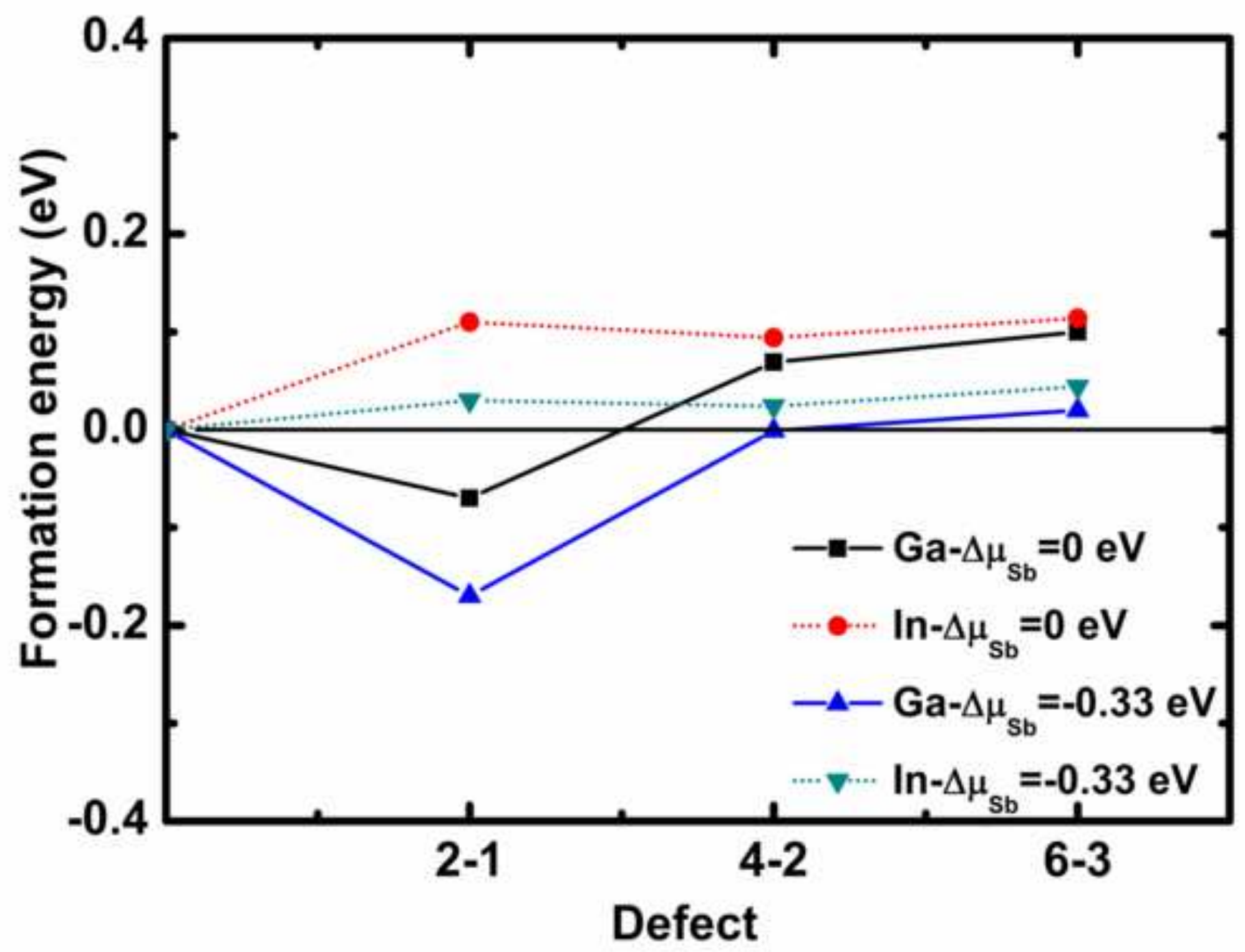


Table 1. Formation energies of different defects at $0 \mathrm{~K}$. Values are given for the chemical potentials in the Co-rich $\left(\Delta \mu_{\mathrm{Sb}}=-0.33 \mathrm{eV}\right)$ and Sb-rich $\left(\Delta \mu_{\mathrm{Sb}}=0 \mathrm{eV}\right)$ limits. The formation energies of the several native defects are also shown in the table for comparison. Only zero charge state defects are listed. The charge state dependence is given in Fig. 3.

\begin{tabular}{c|cc|cc}
\hline \hline $\mathrm{M}$ & \multicolumn{2}{|c|}{$\mathrm{Ga}(\mathrm{eV})$} & \multicolumn{2}{c}{$\mathrm{In}(\mathrm{eV})$} \\
\hline $\mathrm{Defect}$ & $\mathrm{E}\left(\Delta \mu_{\mathrm{Sb}}=0\right)$ & $\mathrm{E}\left(\Delta \mu_{\mathrm{Sb}}=-0.33\right)$ & $\mathrm{E}\left(\Delta \mu_{\mathrm{Sb}}=0\right)$ & $\mathrm{E}\left(\Delta \mu_{\mathrm{Sb}}=-0.33\right)$ \\
\hline $\mathrm{M}_{\mathrm{VF}}$ & 0.594 & 0.594 & 0.471 & 0.471 \\
$\mathrm{M}_{\mathrm{i}}$ & 0.896 & 0.896 & 1.458 & 1.458 \\
$\mathrm{M}_{\mathrm{Sb}}$ & 0.676 & 0.346 & 0.898 & 0.568 \\
$\mathrm{M}_{\mathrm{VF}}-\mathrm{M}_{\mathrm{Sb}}(1-1)$ & 0.436 & 0.271 & 0.534 & 0.369 \\
$2 \mathrm{M}_{\mathrm{VF}}-\mathrm{M}_{\mathrm{Sb}}(2-1)$ & 0.309 & 0.199 & 0.368 & 0.258 \\
$3 \mathrm{M}_{\mathrm{VF}}-\mathrm{M}_{\mathrm{Sb}}(3-1)$ & 0.373 & 0.290 & 0.382 & 0.299 \\
$4 \mathrm{M}_{\mathrm{VF}}-\mathrm{M}_{\mathrm{Sb}}(4-1)$ & 0.414 & 0.348 & 0.398 & 0.332 \\
$5 \mathrm{M}_{\mathrm{VF}}-2 \mathrm{M}_{\mathrm{Sb}}(5-2)$ & 0.397 & 0.303 & 0.363 & 0.269 \\
$4 \mathrm{M}_{\mathrm{VF}}-2 \mathrm{M}_{\mathrm{Sb}}(4-2)$ & 0.314 & 0.204 & 0.359 & 0.249 \\
$6 \mathrm{M}_{\mathrm{VF}}-3 \mathrm{M}_{\mathrm{Sb}}(6-3)$ & 0.337 & 0.227 & 0.352 & 0.242 \\
$\mathrm{M}_{\mathrm{Sb}}-\mathrm{Sb}_{\mathrm{VF}}$ & 0.920 & 0.920 & 1.476 & 1.476 \\
$\mathrm{M}_{\mathrm{Co}}$ & 1.007 & 1.997 & 1.715 & 2.705 \\
$\mathrm{~V}_{\mathrm{Sb}}-\mathrm{M}_{\mathrm{Sb}}$ & 2.678 & 2.078 & 2.777 & 2.117 \\
\hline $\mathrm{Co}_{\mathrm{i}}$ & $0.897(0.8)^{\mathrm{a}}$ & $1.887(2.19)^{\mathrm{a}}$ & & \\
$\mathrm{Sb}_{\mathrm{i}}$ & $2.46^{\mathrm{a}}$ & $2.0{ }^{\mathrm{a}}$ & & \\
$\mathrm{V}_{\mathrm{Co}}$ & $2.89^{\mathrm{a}}$ & $1.5^{\mathrm{a}}$ & & \\
$\mathrm{V}_{\mathrm{Sb}}$ & $2.75^{\mathrm{a}}$ & 3.21 & \\
\hline \hline
\end{tabular}

${ }^{a}$ Park et al., Phys. Rev. B. 81, 085206 (2010). 